\title{
DIFFEOMORPHISM GROUPS OF CONNECTED SUM OF A PRODUCT OF SPHERES AND CLASSIFICATION OF MANIFOLDS
}

SAMUEL OMOLOYE AJALA

\author{
School of Mathematics \\ The Institute for Advanced Study and University of Lagos \\ Princeton, New Jersey 08540 \\ Akoka-Yaba \\ Lagos-Nigeria, West Africa
}

(Received April 21, 1986)

ABSTRACT. In [1] and [2] a classification of a manifold $M$ of the type (n,p, 1) was given where $H_{p}(M)=H_{n-p}(M)=Z$ is the only non-trivial homology groups. In this paper we give a complete classification of manifolds of the type $(n, p, 2)$ and we extend the result to manifolds of type $(n, p, r)$ where $r$ is any positive integer and $p=3,5,6,7 \bmod (8)$.

KEY WORDS AND PHRASES. Pseudo-diffeotopy classes of diffeomorphisms, diffeotopy. 1980 AMS SUBJECT CLASSIFICATION CODE. 57R55

\section{INTRODUCTION.}

In [1] Edward C. Turner worked on a classification of a manifold $M$ of the type $(n, p, r)$ where this means that $M$ is simply connected smooth $n$-manifold and $\mathrm{H}_{\mathrm{p}}(M) \approx \mathrm{H}_{\mathrm{n}-1}(M) \approx \mathbf{z}^{\mathrm{r}}$ the only non-trivial homology groups except for the top and bottom groups. He gave a classification of such manifolds for the case $r=1$ and $p=3,5,6,7(\bmod 8)$. So Turner gave a classification of $M$ of type $(n, p, 1)$ and $p=3,5,6,7(\bmod 8)$. In [2] Hajime Sato independently obtained similar results for $M$ of the type $(n, p, 1)$. The question which naturally follows is: Suppose $r=2,3,4$ and so on, what is the classification of such $M$ ? i.e., what is the classification of $M$ of the type $(n, p, 2),(n, p, 3)$ and so on? In this paper we will study manifolds for the type $(n, p, 2)$ and give its complete classification and then generalize the result to manifolds $M$ of the type (n,p,r) where $r$ is an integer and $p=3,5,6,7(\bmod 8)$.

In $\$ 1$ we prove the following

THEOREM 1.1 Let $M$ be an $n$-dimensional oriented, closed, simply connected manifold of the type $(n, p, 2)$ with $p=3,5,6,7(\bmod 8)$. Then $M$ is diffeomorphic to $\mathrm{s}^{\mathrm{p}} \times \mathrm{D}^{\mathrm{q}+1_{\#}} \mathrm{~s}^{\mathrm{p}} \times \mathrm{D}^{\mathrm{q}+1} \cup \mathrm{s}^{\mathrm{p}} \times \mathrm{D}^{\mathrm{q}+1^{1}} \mathrm{~s}^{\mathrm{p}} \times \mathrm{D}^{\mathrm{q}+1}$ where $\mathrm{n}=\mathrm{p}+\mathrm{q}+1$, \# means connected a $h \quad \partial \quad \partial$ sum along the boundary as defined by Milnor and Karvaire [3] and $h: s^{p} \times s^{q} \# s^{p} \times s^{q} \rightarrow$ $\rightarrow s^{p} \times s^{q} \# s^{p} \times s^{q}$ is a diffeomorphism.

In $\$ 2$ we compute the group $\tilde{\pi}_{0} \operatorname{Diff}\left(s^{p} \times s^{q} \# s^{p} \times s^{q}\right)$ of pseudo-diffeotopy classes of diffeomorphisms of $s^{p} \times s^{q} \# s^{p} \times s^{q} p<q$. 
Let $\mathrm{GL}(2, \mathrm{Z})$ denote the set of $2 \times 2$ unimodular matrices and $\mathrm{H}$ the subgroup of $\mathrm{GL}(2, \mathrm{Z})$ consisting of matrices $\left(\begin{array}{ll}a & b \\ c & d\end{array}\right)$ such that $6=c d=0 \bmod 2$ and $z_{4}$ the subgroup of $G L(2, Z)$ of order 4 generated by $\left(\begin{array}{rr}0 & 1 \\ -1 & 0\end{array}\right)$. We will adopt the notation $M_{p, q}=\operatorname{Diff}\left(s^{p} \times s^{q} \# s^{p} \times s^{q}\right)$ and $M_{p, q}^{+}$the subgroup of $M_{p, q}$ consisting of diffeomorphisms which induce identity map on all homology groups. We will then prove the following

THEOREM 2.1 (i) If $p+q$ is even, then

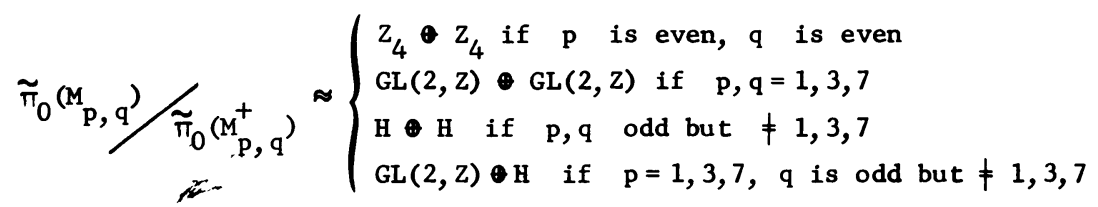

(ii) If $p+q$ is odd then

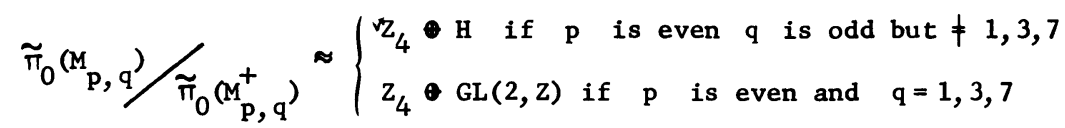

We will further prove the following .

THEOREM 2.15 If $p<q$ and $p=3,5,6,7(\bmod 8)$ the order of the group $\tilde{\pi}_{0}\left(M_{p, q}^{+}\right)$ is twice the order of the group $\pi_{q}(S O(p+1)) \bullet \theta^{p+q+1}$.

In $\$ 3$ we apply the result in $\$ 2$ to prove the following

THEOREM 3.7 Let $M$ be an n-dimensional, smooth, closed, oriented manifold such that $\mathrm{n}=\mathrm{p}+\mathrm{q}+1$ and

$$
H_{i}(M)= \begin{cases}z & i=0, n \\ z \bullet z & i=p, q+1 \\ 0 & \text { elsewhere }\end{cases}
$$

then if $p=3,5,6,7$ (mod 8) the number of differentiable manifolds up to diffeomorphism satisfying the above is equal to twice the order of the group $\pi_{q}(S O(p+1)) \oplus \theta^{p+q+1}$. With induction hypothesis and technique used in $\$ 1$ and $\$ 2$, one can prove the following

THEOREM 3.8 If $M$ is a smooth, closed simply connected manifold of type $(n, p, r)$ where $n=p+q+1$ and $p=3,5,6,7(\bmod 8)$, then the number of differentiable manifolds up to diffeomorphism satisfying the above is equal to

\section{MANIFOLDS OF TYPE $(n, p, r)$}

$$
r \text { times the order of } \pi_{q} s 0(p+1) \bullet \theta^{p+q+1} \text {. }
$$

DEFINITION: Let $M$ be a closed, simply connected $n$-manifold. $M$ is said to be of type $(n, p, r)$ if

$$
H_{i}(M)= \begin{cases}z & \text { if } \quad i=0, n \\ z^{r} & \text { if } i=p, q+1 \\ 0 & \text { elsewhere }\end{cases}
$$

where $\mathrm{n}=\mathrm{p}+\mathrm{q}+1$

We recall from Milnor and Kervaire [3]

DEFINITION: Let $M_{1}$ and $M_{2}$ be $(p+q+1)$-manifolds with boundary and ${ }^{p+q+1}$ 
be half-disc, i.e.,

$$
{ }_{\mathrm{H}}^{\mathrm{p}+\mathrm{q}+1}=\left\{\mathrm{x}=\mathrm{x}_{1}, \mathrm{x}_{2}, \ldots, \mathrm{x}_{\mathrm{p}+\mathrm{q}+1}|| \mathrm{x} \mid \leq 1, \mathrm{x}_{1} \geq 0\right\}
$$

Let $\mathrm{D}^{\mathrm{p}+\mathrm{q}}$ be the subset of $\mathrm{H}^{\mathrm{p}+\mathrm{q}+1}$ for which $\mathrm{x}_{1}=0$. We can choose embeddings

$$
i_{\alpha}:\left(H^{p+q+1}, D^{p+q}\right) \longrightarrow\left(M_{\alpha}, \partial M_{\alpha}\right) \quad \alpha=1,2
$$

so that $i_{2} \cdot i_{1}^{-1}$ reverses orientation. We then form the sum $\left(M_{1}-i_{1}(0)\right)+\left(M_{2}-i_{2}(0)\right)$ by identifying $i_{1}(t u)$ with $i_{2}((1-t) u)$ for $0<t<1 u \in s^{p+q} \cap H^{p+q+1}$. This sum is called the connected sum along the boundary and will be denoted by $M_{1} \# M_{2}$.

REMARK: (1) Notice that the boundary of $M_{1} \# M_{2}$ is $\partial M_{1} \# \partial M_{2}$.

(2) $M_{1} \# M_{2}$ has the homotopy type of $M_{1} \vee M_{2}$ : the union with a single point in common.

THEOREM 1.1 If $M$ is a smooth manifold of type $(n, p, 2)$ where $n=p+q+1$ and $p=3,5,6,7(\bmod 8)$ then there exists a diffeomorphism

$$
h: s^{p} \times s^{q} \# s^{p} \times s^{q} \rightarrow s^{p} \times s^{q} \# s^{p} \times s^{q}
$$

which induce identity on homology such that $M$ is diffeomorphic to

$$
\mathrm{s}^{\mathrm{p}} \times \mathrm{D}^{\mathrm{q}+1} \# \mathrm{~s}^{\mathrm{p}} \times \mathrm{D}_{\mathrm{h}}^{\mathrm{q}+1} \underset{\mathrm{U}}{\cup} \mathrm{s}^{\mathrm{p}} \times \mathrm{D}^{\mathrm{q}+1} \# \mathrm{~s}^{\mathrm{p}} \times \mathrm{D}^{\mathrm{q}+1}
$$

PROOF: Let $\left\{M, \lambda_{1}, \lambda_{2}\right\}$ be a manifold of type $(n, p, 2)$ and $\lambda_{1}, \lambda_{2}$ represent the generators of the first and second summands of $H_{p}(M) \approx Z \oplus Z$. We can choose embeddings $\varphi_{i}: s^{P} \rightarrow M$ so as to represent the homology class $\lambda_{i} i=1,2$. Since $p<q$, two homotopic embeddings are isotopic. Let $\left.\alpha_{i} \in \pi_{p-1} s 0(q+1)\right)$ be the characteristic class of the embedded sphere $s^{p}$, since $p=3,5,6,7$ (mod 8), the normal bundle of the embedded sphere is trivial. It follows that $\varphi_{i}$ extends to an embedding $\varphi_{i}^{\prime}: S^{P} \times D^{q+1} \rightarrow M$ such that its homology class is $\lambda_{i} \cdot$ Then we can form a connected sum along the boundary of the two embedded copies of $s^{p} \times D^{q+1}$ to get $s^{p} \times D^{q+1} \# s^{p} \times D^{q+1}$. We then have an embedding $i: s^{p} \times D^{q+1} \# s^{p} \times D^{q+1} \rightarrow M$

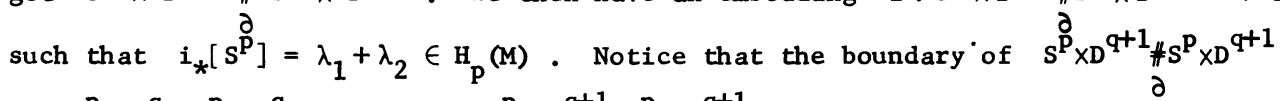
is $S^{p} \times S^{q} \# S^{p} \times S^{q}$ and since $S^{p} \times D^{q+1} \# s^{p} \times D^{q+1}$ has the homotopy type of $s^{p} \times D^{q+1} \vee s^{p} \times D^{q+1}$ then it is easy to see that

$$
H_{i}\left(S^{p} \times D^{q+1} \# S^{p} \times D^{q+1}\right)=\left\{\begin{array}{ll}
z & \text { for } i=0 \\
z \oplus z & \text { for } i=p
\end{array}\right. \text {. }
$$

It is also easy to see that

$$
H_{i}\left(M-\operatorname{Int}\left(S^{p} \times D^{q+1} \# S^{p} \times D^{q+1}\right)\right)= \begin{cases}z & \text { for } i=0 \\ z \in z & \text { for } i=p\end{cases}
$$

Now since $S^{p} \times D^{q+1}$ is a trivial disc bundle over $s^{p}$ then it has cross sections; hence, there exists orientation reversing diffeomorphism of $s^{p} \times D^{q+1} \# s^{p} \times D^{q+1}$ onto itse1f. Thus there exists an orientation reversing embedding

$$
j: s^{p} \times D^{q+1} \# s^{p} \times D^{q+1} \rightarrow M-\operatorname{Int}\left(s^{p} \times D_{\partial}^{q+1} \# s^{p} \times D^{q+1}\right)
$$


such that $j_{*}\left[S^{P}\right]=\lambda_{1}+\lambda_{2}$ and in fact this embedding is a homotopy equivalence. It follows by [4, Thm. 4.1] that $S^{p} \times D^{q+1} \# s^{p} \times D^{q+1}$ is diffeomorphic to $M-\operatorname{Int}\left(S^{P} \times D^{q+1} \# s^{p} \times D^{q+1}\right)$. Consequently, it follows that $M$ is diffeomorphic to $s^{p} \times D^{q+1} \# S^{p} \times D^{q^{q}+1} \cup S^{p} \times D^{q+1} \# s^{p} \times D^{q+1}$ for an orientation preserving diffeomorphism $h: s^{p} \times s^{q} \# s^{p} \times s^{q} \stackrel{h}{\rightarrow} s^{p} \times s^{q}{ }^{\partial} s^{p} \times s^{q}$. From the embeddings in the proof, it is clear that $h$ induce identity on homology.

2. THE GROUP $\tilde{\pi}_{0} \operatorname{Diff}\left(s^{p} \times s^{q} \# s^{p} \times s^{q}\right)$

For convenience, we adopt the notation $M_{p, q}=\operatorname{Diff}\left(s^{p} \times s^{q} \# s^{p} \times s^{q}\right)$ and $M_{p, q}^{+}$ the subset of $M_{p, q}$ consisting of diffeomorphisms of $s^{p} \times s^{q} \# s^{p} \times s^{q}$ which induce identity on all homology groups.

DEFINITION: Let $M$ be an oriented smooth manifold. Diff(M) is the group of orientation preserving diffeomorphisms of $M$. Let $f, g \in \operatorname{Diff}(M), \quad f$ and $g$ are said to be pseudo-diffeotopic if there exists a diffeomorphism $H$ of $M \times I$ such that $H(x, 0)=(f(x), 0)$ and $H(x, 1)=(g(x), 1)$ for all $x \in M$. The pseudo-diffeotopy class of diffeomorphisms of $M$ is denoted by $\tilde{\pi}_{0}$ (Diff $M$ ). We wish to compute $\tilde{\pi}_{0}^{\prime}\left(M_{p, q}\right)$ for $p<q$. If $f, \in M_{p, q}$ then $f$ induces an automorphism $f_{*}: H_{*}\left(s^{p} \times s^{q} \# s^{p} \times s^{q}\right) \rightarrow H_{*}\left(s^{p} \times s^{q} \# s^{p} \times s^{q}\right)$

of homology groups of $S^{p} \times S^{q} \# S^{p} \times S^{q}$. Since pseudo-diffeotopic diffeomorphisms induce equal automorphism on homology then we have a well-defined homomorphism

$$
\Phi: \tilde{\pi}_{0}\left(M_{p, q}\right) \rightarrow \text { Auto }\left(H_{*}\left(s^{p} \times s^{q} \# s^{p} \times s^{q}\right)\right)
$$

where Auto $\left(\mathrm{H}_{*}\left(\mathrm{~S}^{\mathrm{p}} \times \mathrm{S}^{\mathrm{q}} \# \mathrm{~S}^{\mathrm{p}} \times \mathrm{S}^{\mathrm{q}}\right)\right.$ denotes the group of dimension preserving automorphisms of $\mathrm{H}_{*}\left(\mathrm{~S}^{\mathrm{P}} \times \mathrm{S}^{\mathrm{q}} \# \mathrm{~S}^{\mathrm{P}} \times \mathrm{S}^{\mathrm{q}}\right)$.

THEOREM 2.1 (i) If $\mathrm{p}+\mathrm{q}$ is even then

$$
\Phi\left(\tilde{\pi}_{0}\left(M_{p, q}\right)\right)= \begin{cases}\mathbf{z}_{4} \oplus \mathbf{z}_{4} & \text { if } \mathrm{p}, \mathrm{q} \text { are even } \\ \mathrm{GL}(2, \mathrm{Z}) \oplus \mathrm{GL}(2, \mathrm{Z}) & \text { if } \mathrm{p}, \mathrm{q} \text { are } 1,3,7 \\ \mathrm{H} \bullet \mathrm{H} & \text { if } \mathrm{p}, \mathrm{q} \text { are odd but } \neq 1,3,7 \\ \mathrm{GL}(2, \mathrm{z}) \bullet \mathrm{H} & \text { if } \mathrm{p}=1,3,7 \text { and } \mathrm{q} \text { is odd but } \neq 1,3,7\end{cases}
$$

The following propositions give the proof of Theorem 2.1.

PROPOSITION 2.1 If $\mathrm{p}+\mathrm{q}$ is even, $\mathrm{p}$ is even, then

$$
\Phi\left(\tilde{\pi}_{0}\left(M_{p, q}\right)\right)=z_{4} \oplus z_{4} \text {. }
$$

PROOF: Since $p+q$ is even and $p$ is even then $q$ must also be even. We have

$$
H_{i}\left(S^{p} \times S^{q} \# S^{p} \times S^{q}\right)=\left\{\begin{array}{ll}
z & \text { if } i=0, p+q \\
z \oplus z & \text { if } i=p \text { or } q \\
0 & \text { elsewhere }
\end{array}\right. \text {. }
$$

Generators of $\mathrm{H}_{0}\left(\mathrm{~S}^{\mathrm{p}} \times \mathrm{S}^{\mathrm{q}} \# \mathrm{~S}^{\mathrm{p}} \times \mathrm{S}^{\mathrm{q}}\right)$ and $\mathrm{H}_{\mathrm{p}+\mathrm{q}}\left(\mathrm{S}^{\mathrm{p}} \times \mathrm{S}^{q} \# \mathrm{~S}^{\mathrm{p}} \times \mathrm{S}^{\mathrm{q}}\right)$ are mapped to the same generators but $H_{p}\left(S^{p} \times S^{q} \# S^{p} \times S^{q}\right)=z \bullet Z$. If $f \in M_{p}, q$, we shall denote by $\Phi(f)_{p}$ the automorphism $f_{*}: H_{p}\left(s^{p} \times s^{q} \# s^{p} \times s^{q}\right) \rightarrow H_{p}\left(s^{p} \times s^{q} \# s^{p} \times s^{q}\right)$ induced by the image $f$ under $\Phi$ in dimension $p$. Then $\Phi(f)_{p}=f_{*}: z \bullet z \rightarrow z \bullet z$ is the induced 
automorphism. If $e_{1}, e_{2}$ are the generators of the first and second summand of $\mathrm{H}_{\mathrm{p}}\left(\mathrm{S}^{\mathrm{P}} \times \mathrm{S}^{\mathrm{q}} \# \mathrm{~S}^{\mathrm{p}} \times \mathrm{S}^{\mathrm{q}}\right)$ if $\circ$ denotes the intersection then $\mathrm{e}_{1} \circ \mathrm{e}_{1}=0, \mathrm{e}_{2} \circ \mathrm{e}_{2}=0$, $e_{1} \circ e_{2}=1$ and $e_{2} \circ e_{1}=-1$. Let $\left(\begin{array}{ll}a_{1} & a_{2} \\ a_{3} & a_{4}\end{array}\right) \in G L(2, z)$, if $\Phi(f)$ takes $e_{1}, e_{2}$ to $e_{1}^{\prime}, e_{2}^{\prime}$ respectively then $e_{1}^{\prime}=a_{1} e_{1}+a_{2} e_{2}$ and $e_{2}^{\prime}=a_{3} e_{1}+a_{4} e_{2}$ then

$$
\begin{aligned}
e_{1}^{\prime} \circ e_{1}^{\prime} & =\left(a_{1} e_{1}+a_{2} e_{2}\right) \cdot\left(a_{1} e_{1}+a_{2} e_{2}\right) \\
& =a_{1} a_{1} e_{1} \cdot e_{1}+a_{1} a_{2} e_{1} \cdot e_{2}+a a_{1} e_{2} \cdot e_{1}+a_{2} a_{2} e_{2} \cdot e \\
& =a_{1} a_{2} e_{1} \cdot e_{2}+a_{2} a_{1} e_{2} \cdot e_{1}=a_{1} a_{2}-a_{1} a_{2}=0 .
\end{aligned}
$$

Similarly $e_{2}^{\prime} \cdot e_{2}^{\prime}=0$

but $e_{1}^{\prime} \cdot e_{2}^{\prime}=\left(a_{1} e_{1}+a_{2} e_{2}\right) \circ\left(a_{3} e_{1}+a_{4} e_{2}\right)$

$=a_{1} a_{3} e_{1} \circ e_{1}+a_{1} a_{4} e_{1} \circ e_{2}+a_{2} a_{3} e_{2} \circ e_{1}+a_{2} a_{4} e_{2} \circ e_{2}$

$=a_{1} a_{4}-a_{2} a_{3}=1$ since $G L(2, Z)$ is unimodular.

$e_{2}^{\prime} \cdot e_{1}^{\prime}=\left(a_{3} e_{1}+a_{4} e_{2}\right) \cdot\left(a_{1} e_{1}+a_{2} e_{2}\right)=a_{3} a_{1} e_{1} \circ e_{1}+a_{3} a_{2} e_{1} \circ e_{2}+a_{4} a_{1} e_{2} \circ e_{1}$ $+a_{4} a_{2} e_{2} \circ e_{2}=a_{3} a_{2}-a_{4} a_{1}=-1$

hence for $p$ even $\Phi(f)_{p}$ is an element of a subgroup of $G L(2, z)$ generated by

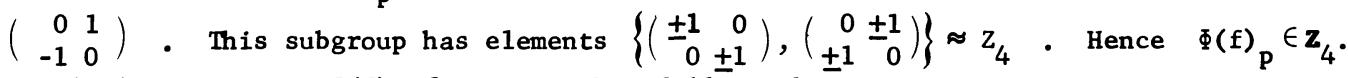
Similarly for $i=q \quad \Phi(f)_{q} \in \mathbf{z}_{4}$, it then follows that

$$
\Phi\left(\tilde{\pi}_{0}\left(M_{p, q}\right)\right) \subset \mathbf{z}_{4} \oplus \mathbf{z}_{4} \text {. }
$$

We now show that $z_{4} \oplus \mathbf{z}_{4} \subset \Phi\left(\tilde{\pi}_{0}\left(M_{p, q}\right)\right)$. We need to show that the generators of $\mathbf{z}_{4} \oplus \mathbf{z}_{4}$ can be realized as the image of $\Phi$. We shall adopt the notation $\left(S^{p} \times S^{q}\right)_{1} \#\left(S^{p} \times S^{q}\right)_{2}$ where the subscripts 1 and 2 denote the first and second summands of $\mathrm{s}^{\mathrm{p}} \times \mathrm{S}^{\mathrm{q}} \# \mathrm{~s}^{\mathrm{p}} \times \mathrm{S}^{\mathrm{q}}$ and let $\mathrm{R}_{\mathrm{p}}$ and $\mathrm{R}_{\mathrm{q}}$ be reflections of $\mathrm{s}^{\mathrm{p}}$ and $\mathrm{s}^{\mathrm{q}}$ respectively. If $\left(x_{1}, y_{1}\right) \in\left(s^{p} \times s^{q}\right)_{1}$ and $\left(x_{2}, y_{2}\right) \in\left(s^{p} \times s^{q}\right)_{2}$, we define $f \in M_{p, q}$

$$
\begin{aligned}
& f\left(x_{1}, y_{1}\right)=\left(R_{p}\left(x_{2}\right), R_{q}\left(y_{2}\right)\right) \\
& f\left(x_{2}, y_{2}\right)=\left(x_{1}, y_{1}\right)
\end{aligned}
$$

In other words $f\left(\left(x_{1}, y_{1}\right)\left(x_{2}, y_{2}\right)\right)=\left(\left(R_{p}\left(x_{2}\right), R_{q}\left(y_{2}\right)\right),\left(x_{1}, y_{1}\right)\right)$

$$
\left(x_{1}, y_{1}\right) \in\left(s^{p} \times s^{q}\right)_{1} \text { and }\left(x_{2}, y_{2}\right) \in\left(s^{p} \times s^{q}\right)_{2} \text {. }
$$

For $\Phi(f)_{p} \in$ Auto $H_{p}(M p, q)$, if $e_{1}, e_{2}$ are the generators of the first and second summands of $H_{p}\left(S^{p} \times S^{q} \# S^{p} \times S^{q}\right)=z \oplus z$ since $f$ takes $x_{1}$ to $R_{p}\left(x_{2}\right)$ and $f$ takes $x_{2}$ to $x_{1}$, then it is easily seen that $\Phi(f)_{p}\left(e_{1}\right)=-e_{2}$ and $\Phi(f)_{p}\left(e_{2}\right)=e_{1}$. Hence $e_{1}^{\prime}=-e_{2}$ and $e_{2}^{\prime}=e_{1}$ and so $e_{1}^{\prime} \circ e_{1}^{\prime}=-e_{2} \circ-e_{2}=0$, $e_{2}^{\prime} \circ e_{2}^{\prime}=e_{1} \circ e_{1}=0, e_{1}^{\prime} \circ e_{2}^{\prime}=-e_{2} \circ e_{1}=1$ and $e_{2}^{\prime} \circ e_{1}^{\prime}=e_{1} \circ-e_{2}=-1$. Hence $\Phi$ maps $f$ in dimension $p$ to $\left(\begin{array}{rr}0 & 1 \\ -1 & 0\end{array}\right)$ which generates $z_{4}$. Similar argument shows that $\Phi$ maps $f$ in dimension $q$ to $\left(\begin{array}{rr}0 & 1 \\ -1 & 0\end{array}\right)$ which generates $z_{4} \cdot$ Then $\Phi$ maps onto $\mathrm{Z}_{4} \oplus \mathrm{Z}_{4}$ hence the proof.

PROPOSITION 2.2 If $p+q$ is even but $p, q=1,3,7$ then $\Phi\left(\tilde{\pi}_{0}(M, q)\right)=$ $=\mathrm{GL}(2, \mathrm{Z}) \oplus \mathrm{GL}(2, \mathrm{Z})$.

PROOF : From [5, Appendix B] and [6] one sees that $\mathrm{GL}(2, \mathrm{Z})$ is generated by $\left(\begin{array}{rr}0 & 1 \\ -1 & 0\end{array}\right)$ and $\left(\begin{array}{ll}1 & 1 \\ 0 & 1\end{array}\right)$. Since $p, q=1,3,7$ it follows by $[7, \$ 1]$ that there exist maps $f: s^{p} \rightarrow S O(p+1)$ and $g: s^{q} \rightarrow S O(q+1)$ such that $f$ and $g$ have index +1 . 
We then define $h \in M_{p, q}$

$$
\begin{array}{ll}
h\left(x_{1}, y_{1}\right)=\left(x_{1}, y_{1}\right) & \left(x_{1}, y_{1}\right) \in\left(s^{p} \times s^{q}\right)_{1} \\
h\left(x_{2}, y_{2}\right)=\left(f\left(x_{1}\right) \cdot x_{2}, g\left(y_{1}\right) \cdot y_{2}\right) & \left(x_{2}, y_{2}\right) \in\left(s^{p} \times s^{q}\right)_{2}
\end{array}
$$

i.e., $h\left(\left(x_{1}, y_{1}\right),\left(x_{2}, y_{2}\right)\right)=\left(\left(x_{1}, y_{1}\right),\left(f\left(x_{1}\right) \cdot x_{2}, g\left(y_{1}\right) \cdot y_{2}\right)\right)$

Since $f$ has index +1 and $h$ takes $x_{1}$ to $x_{1}$ and $x_{2}$ to $f\left(x_{1}\right) \cdot x_{2}$ then it follows by an easy application of [7, Prop. 1.2] or [6, Prop. 2.3] that $\Phi(\mathrm{h}){ }_{\mathrm{p}}$ is $\left(\begin{array}{ll}1 & 1 \\ 0 & 1\end{array}\right)$ also since $g$ has index +1 and $h$ takes $y_{1}$ to $y_{1}$ and $y_{2}$ to $g\left(y_{1}\right) \cdot y_{2}$ then $\Phi(h)_{q}$ is $\left(\begin{array}{ll}1 & 1 \\ 0 & 1\end{array}\right)$. Hence $\Phi$ maps $h$ to $\left\{\left(\begin{array}{ll}1 & 1 \\ 0 & 1\end{array}\right),\left(\begin{array}{ll}1 & 1 \\ 0 & 1\end{array}\right)\right\}$. We now define $\alpha \in M_{p, q}$ by

$$
\begin{array}{ll}
\alpha\left(x_{1}, y_{1}\right)=\left(R_{p}\left(x_{2}\right), R_{q}\left(y_{2}\right)\right) & \left(x_{1}, y_{1}\right) \in\left(s^{p} \times s^{q}\right)_{1} \\
\alpha\left(x_{2}, y_{2}\right)=\left(x_{1}, y_{1}\right) & \left(x_{2}, y_{2}\right) \in\left(s^{p} \times s^{q}\right)_{2}
\end{array}
$$

i.e., $\alpha\left(\left(x_{1}, y_{1}\right),\left(x_{2}, y_{2}\right)\right)=\left(\left(R_{p}\left(x_{2}\right), R_{q}\left(y_{2}\right)\right),\left(x_{1}, y_{1}\right)\right)$

Since $\propto$ takes $x_{1}$ to $R_{p}\left(x_{2}\right)$ and $x_{2}$ to $x_{1}$ it follows from Proposition 2.1 that $\Phi(\alpha)_{\mathrm{p}}$ is $\left(\begin{array}{rr}0 & 1 \\ -1 & 0\end{array}\right)$ and by similar reasoning $\Phi(\alpha)_{\mathrm{q}}$ is $\left(\begin{array}{rr}0 & 1 \\ -1 & 0\end{array}\right)$. This means that $\Phi$ maps $\alpha$ to $\left\{\left(\begin{array}{rr}0 & 1 \\ -1 & 0\end{array}\right),\left(\begin{array}{rr}0 & 1 \\ -1 & 0\end{array}\right)\right\}$. Since $\mathrm{GL}(2, \mathrm{Z})$ is generated by $\left(\begin{array}{ll}1 & 1 \\ 0 & 1\end{array}\right)$ and $\left(\begin{array}{rr}0 & 1 \\ -1 & 0\end{array}\right)$ then it follows that for $p, q=1,3,7$

$$
\Phi\left(\tilde{\pi}_{0}\left(M_{p, q}\right)\right) \approx G L(2, z) \oplus \mathrm{GL}(2, z) \text {. }
$$

PROPOSITION 2.3 If $\mathrm{p}+\mathrm{q}$ is even but $\mathrm{p}$ and $\mathrm{q}$ are odd but $\mathrm{p}, \mathrm{q} \neq 1,3,7$, then $\Phi\left(\tilde{\pi}_{0}\left(M_{p, q}\right)\right) \approx H \oplus H$.

PROOF: By using Proposition 2.1 and [8, Lemma 5] it is enough to produce a diffeomorphism in $M_{p, q}$ whose image under $\Phi$ is $\left(\begin{array}{ll}1 & 2 \\ 0 & 1\end{array}\right)$ in each of the dimensions $\mathrm{p}$ and $\mathrm{q}$. This is because $\left(\begin{array}{rr} \pm 1 & 0 \\ 0 & \pm 1\end{array}\right),\left(\begin{array}{rr}0 & \pm 1 \\ \pm 1 & 0\end{array}\right),\left(\begin{array}{ll}1 & 2 \\ 0 & 1\end{array}\right)$ generate $\mathrm{H}$. However $\left(\begin{array}{rr} \pm 1 & 0 \\ 0 & \pm 1\end{array}\right)$ is trivially the image under $\Phi$ of identity map and reflections on each coordinate while $\left(\begin{array}{r}0 \\ \pm 1 \\ \pm\end{array}\right)$ is by Proposition 2.1 the image under $\Phi$ of an element of $M_{p, q}$. However, there exists a map $\alpha: s^{p} \rightarrow s 0(p+1)$ of index 2 by [8] so also is a map $\beta: s^{q} \rightarrow s o(q+1)$ of index 2 and then we can define $f \in M_{p, q}$ thus.

$$
\begin{array}{ll}
f\left(x_{1}, y_{1}\right)=\left(x_{1}, y_{1}\right) & \left(x_{1}, y_{1}\right) \in\left(s^{p} \times s^{q}\right)_{1} \\
f\left(x_{2}, y_{2}\right)=\left(\alpha\left(x_{1}\right) \cdot x_{2}, \beta\left(y_{1}\right) \cdot y_{2}\right) & \left(x_{2}, y_{2}\right) \in\left(s^{p} \times s^{q}\right)_{2}
\end{array}
$$

i.e., $f\left(\left(x_{1}, y_{1}\right),\left(x_{2}, y_{2}\right)\right)=\left(\left(x_{1}, y_{1}\right),\left(\alpha\left(x_{1}\right) \cdot x_{2}, \beta\left(y_{1}\right) \cdot y_{2}\right)\right)$.

It easily follows that since $f$ takes $x_{1}$ to $x_{1}$ and takes $x_{2}$ to $\alpha\left(x_{1}\right) \cdot x_{2}$ with $\alpha$ having index 2 then it follows by applying [7, Lemma 5] that $\Phi(f)_{p}$ is $\left(\begin{array}{ll}1 & 2 \\ 0 & 1\end{array}\right)$. Similar argument shows that $\Phi(f)_{q}$ is $\left(\begin{array}{ll}1 & 2 \\ 0 & 1\end{array}\right)$; hence $\Phi\left(\pi_{0}\left(M_{p, q}\right)\right) \approx H \oplus H$. PROPOSITION 2.4 If $p+q$ is even, $p=1,3,7$ but $q$ is odd and $\neq 1,3,7$ then $\Phi\left(\tilde{\pi}_{0}\left(M_{p, q}\right)\right)=G L(2, z) \oplus H$.

PROOF : $\left\{\left(\begin{array}{rr}0 & 1 \\ -1 & 0\end{array}\right),\left(\begin{array}{ll}1 & 1 \\ 0 & 1\end{array}\right)\right\}$ generates $G L(2, Z)$ while $\left\{\left(\begin{array}{rr}0 & 1 \\ -1 & 0\end{array}\right),\left(\begin{array}{ll}1 & 2 \\ 0 & 1\end{array}\right)\right\}$ generates $H$, since $q \neq 1,3,7$ and by [8] there exists $\alpha: s^{q} \rightarrow s 0(q+1)$ of index 2 . If $R_{p}$ is reflection of $s^{p}$ then we define $h \in M_{p, q}$ 


$$
\begin{array}{ll}
h\left(x_{1}, y_{1}\right)=\left(R_{p}\left(x_{2}\right), y_{1}\right) & \left(x_{1}, y_{1}\right) \in\left(s^{p} \times s^{q}\right)_{1} \\
h\left(x_{2}, y_{2}\right)=\left(x_{1}, \alpha\left(y_{1}\right) \cdot y_{2}\right) & \left(x_{2}, y_{2}\right) \in\left(s^{p} \times s^{q}\right)_{2}
\end{array}
$$

Since $h$ takes $x_{1}$ to $R_{p}\left(x_{2}\right)$ and takes $x_{2}$ to $x_{1}$ it follows by Proposition 2.1 that $\Phi(h)_{p}=\left(\begin{array}{rr}0 & 1 \\ -1 & 0\end{array}\right) ;$ similarly $h$ takes $y_{1}$ to $y$, and $y_{2}$ to $\alpha\left(y_{1}\right) \cdot y_{2}$ and since $\alpha$ has index 2, it follows that $\Phi(\mathrm{h})_{q}=\left(\begin{array}{ll}1 & 2 \\ 0 & 1\end{array}\right)$.

Now if $R_{q}$ is a reflection on $S^{q}$ and $\beta: S^{p} \longrightarrow S_{p+1}$ is of index +1 then we define $f \in M_{p, q}$

$$
\begin{array}{ll}
f\left(x_{1}, y_{1}\right)=\left(x_{1}, R_{q}\left(y_{2}\right)\right) & \left(x_{1}, y_{1}\right) \in\left(S^{p} \times S^{q}\right)_{1} \\
f\left(x_{2}, y_{2}\right)=\left(\beta\left(x_{1}\right) \cdot x_{2}, y_{1}\right) & \left(x_{2}, y_{2}\right) \in\left(S^{p} \times S^{q}\right)_{2}
\end{array}
$$

then it is easy to see that $\Phi(f)_{p}=\left(\begin{array}{ll}1 & 1 \\ 0 & 1\end{array}\right)$ and $\Phi(f)_{q}=\left(\begin{array}{rr}0 & 1 \\ -1 & 0\end{array}\right)$ so the image of $h$ under $\Phi$ is $\left\{\left(\begin{array}{rr}0 & 1 \\ -1 & 0\end{array}\right),\left(\begin{array}{ll}1 & 2 \\ 0 & 1\end{array}\right)\right\}$ and the image of $f$ under $\Phi$ is $\left\{\left(\begin{array}{rr}0 & 1 \\ -1 & 0\end{array}\right),\left(\begin{array}{ll}1 & 1 \\ 0 & 1\end{array}\right)\right\}$ and since $\left\{\left(\begin{array}{rr}0 & 1 \\ -1 & 0\end{array}\right),\left(\begin{array}{ll}1 & 2 \\ 0 & 1\end{array}\right)\right\}$ generate $H$ and $\left\{\left(\begin{array}{rr}0 & 1 \\ -1 & 0\end{array}\right),\left(\begin{array}{ll}1 & 1 \\ 0 & 1\end{array}\right)\right\}$ generate GL(2, Z) then it follows that $\Phi\left(\pi_{0}\left(M_{p, q}\right)\right) \approx G L(2, Z) \oplus H$. Hence the proof.

REMARK. For $p$ odd but $\neq 1,3,7$ and $q=1,3,7$, we have the same result as , above using the same method but since by assumption $p<q$ only one dimension (consequently one manifold) comes in here, viz $p=5, q=7$, i.e., $s^{5} \times s^{7} \# s^{5} \times s^{7}$.

Combination of Propositions 2.1, 2.2, 2.3, and 2.4 proves Theorem 2.1(i).

PROPOSITION 2.5 Suppose $p+q$ is odd and $p$ is even and $q$ odd $\neq 1,3,7$ then $\Phi\left(\tilde{\pi}_{0}\left(M_{p, q}\right)\right) \approx \boldsymbol{z}_{4} \oplus \mathrm{H}$.

PROOF: Since $\left(\begin{array}{rr}0 & 1 \\ -1 & 0\end{array}\right)$ generate $Z_{4}$ and $\left\{\left(\begin{array}{rr}0 & 1 \\ -1 & 0\end{array}\right),\left(\begin{array}{ll}1 & 2 \\ 0 & 1\end{array}\right)\right\}$ generate $H$, then we on1y need to find the diffeomorphism in $M_{p, q}$ that $\Phi$ maps to these generators. Similar to Proposition 2.4, we define $f \stackrel{\mathrm{P}}{\epsilon}_{\mathrm{M}}^{\mathrm{q}}, \mathrm{q}$ by

$$
\begin{array}{ll}
f\left(x_{1}, y_{1}\right)=\left(R_{p}\left(x_{2}, y_{1}\right)\right. & \left(x_{1}, y_{1}\right) \in\left(s^{p} \times s^{q}\right)_{1} \\
f\left(x_{2}, y_{2}\right)=\left(x_{1}, \alpha\left(y_{1}\right) \cdot y_{2}\right) & \left(x_{2}, y_{2}\right) \in\left(s^{p} \times s^{q}\right)_{2}
\end{array}
$$

where $R_{p}$ is the reflection on $s^{p}$ and $\alpha: s^{q} \rightarrow S_{q+1}$ is of index 2 which exists by [3] since $q \neq 1,3,7$. It then follows that $\Phi(f){ }_{p}=\left(\begin{array}{rr}0 & 1 \\ -1 & 0\end{array}\right)$ and $\left.\Phi(f)\right)_{q}=\left(\begin{array}{ll}1 & 2 \\ 0 & 1\end{array}\right)$. Also we define $g \in M_{p, q}$ thus

$$
\begin{aligned}
& g\left(x_{1}, y_{1}\right)=\left(x_{1}, R_{q}\left(y_{2}\right)\right) \quad\left(x_{1}, y_{1}\right) \in\left(s^{p} \times s^{q}\right)_{1},\left(x_{2}, y_{2}\right) \in\left(s^{p} \times s^{q}\right)_{2} \\
& g\left(x_{2}, y_{2}\right)=\left(x_{2}, y_{1}\right)
\end{aligned}
$$$$
\text { i.e., } g\left(\left(x_{1}, y_{1}\right),\left(x_{2}, y_{2}\right)\right)=\left(\left(x_{1}, R_{q}\left(y_{2}\right)\right),\left(x_{2}, y_{1}\right)\right)
$$

where $R_{q}$ is the reflection on $s^{q}$. Since $g$ takes $x_{1}$ to $x_{1}$ and $x_{2}$ to $x_{2}$ then $\Phi(g)_{p}=$ identity $=\left(\begin{array}{ll}1 & 0 \\ 0 & 1\end{array}\right)$ and since $g$ takes $y_{1}$ to $R_{q}\left(y_{2}\right)$ and $y_{2}$ to $y_{1}$ it follows that by applying Proposition 2.1, $\Phi(\mathrm{g})_{\mathrm{q}}=\left(\begin{array}{rr}0 & 1 \\ -1 & 0\end{array}\right)$. Hence $\mathrm{f}$ is mapped by $\Phi$ to $\left\{\left(\begin{array}{rr}0 & 1 \\ -1 & 0\end{array}\right),\left(\begin{array}{ll}1 & 2 \\ 0 & 1\end{array}\right)\right\}$ while $g$ is mapped by $\Phi$ to $\left\{\left(\begin{array}{ll}1 & 0 \\ 0 & 1\end{array}\right),\left(\begin{array}{rr}0 & 1 \\ -1 & 0\end{array}\right)\right\}$ and since these matrices generate $\mathrm{H}$ and $\mathrm{Z}_{4}$ respectively then it follows that $\Phi\left(\tilde{\pi}_{0}\left(\mathrm{M}_{\mathrm{p}, \mathrm{q}}\right)\right) \approx \mathrm{Z}_{4} \oplus \mathrm{H}$.

PROPOSITION 2.6 Suppose $\mathrm{p}+\mathrm{q}$ is odd and $\mathrm{p}$ is even $\mathrm{q}$ is odd and $=1,3,7$. Then $\Phi\left(\tilde{\pi}_{0}\left(M_{p, q}\right)\right) \approx z_{4} \oplus \mathrm{GL}(2, z)$. 
PROOF: Again since $q=1,3,7$ by [6, Prop. 2.4] there exists a map $\alpha: s^{q} \rightarrow S_{q+1}$ of index 1. Since $\left(\begin{array}{rr}0 & 1 \\ -1 & 0\end{array}\right)$ generates $Z_{4}$ and $\left\{\left(\begin{array}{ll}1 & 1 \\ 0 & 1\end{array}\right),\left(\begin{array}{rr}0 & 1 \\ -1 & 0\end{array}\right)\right\}$ generate $\operatorname{GL}(2, z)$ we define elements of $M_{p, q}$ that are mapped onto these generators. Let $h \in M_{p, q}$ be defined thus

$$
\begin{array}{lll}
h\left(x_{1}, y_{1}\right)=\left(R_{p}\left(x_{2}\right), y_{1}\right) & \text { where } & \left(x_{1}, y_{1}\right) \in\left(s^{p} \times s^{q}\right)_{1} \\
h\left(x_{2}, y_{2}\right)=\left(x_{1}, \alpha\left(y_{1}\right) \cdot y_{2}\right) & \left(x_{2}, y_{2}\right) \in\left(s^{p} \times s^{q}\right)_{2}
\end{array}
$$

i.e., $\left.h\left(x_{1}, y_{1}\right),\left(x_{2}, y_{2}\right)\right)=\left(\left(R_{p}\left(x_{2}\right), y_{1}\right),\left(x_{1}, \alpha\left(y_{1}\right)-y_{2}\right)\right)$

where $R_{p}$ is the reflection of $S^{P}$. Then it is easy to see that $\Phi(h){ }_{p}=\left(\begin{array}{rr}0 & 1 \\ -1 & 0\end{array}\right)$ while $\Phi(\mathrm{h})_{\mathrm{q}}=\left(\begin{array}{ll}1 & 1 \\ 0 & 1\end{array}\right)$. Also one can define $f \in M_{\mathrm{p}, \mathrm{q}}$ as

$$
\begin{aligned}
& f\left(x_{1}, y_{1}\right)=\left(x_{1}, R_{q}\left(x_{2}\right)\right) \text { where }\left(x_{1}, y_{1}\right) \in\left(s^{p} \times s^{q}\right)_{1},\left(x_{2}, y_{2}\right) \in\left(s^{p} \times s^{q}\right)_{2} \\
& f\left(x_{2}, y_{2}\right)=\left(x_{2}, y_{1}\right)
\end{aligned}
$$

i.e., $f\left(\left(x_{1}, y_{1}\right),\left(x_{2}, y_{2}\right)\right)=\left(\left(x_{1}, R_{q}\left(y_{2}\right)\right),\left(x_{2}, y_{1}\right)\right)$ where $R_{q}$ is a reflection of $s^{q}$ and so it is easily seen that $\Phi(f)_{p}=\left(\begin{array}{ll}1 & 0 \\ 0 & 1\end{array}\right)$ while $\Phi(f)_{q}=\left(\begin{array}{rr}0 & 1 \\ -1 & 0\end{array}\right)$ so h is mapped by $\Phi$ to $\left\{\left(\begin{array}{rr}0 & 1 \\ -1 & 0\end{array}\right),\left(\begin{array}{ll}1 & 1 \\ 0 & 1\end{array}\right)\right\}$ while $\mathrm{f}$ is mapped by $\Phi$ to $\left\{\left(\begin{array}{ll}1 & 0 \\ 0 & 1\end{array}\right),\left(\begin{array}{rr}0 & 1 \\ -1 & 0\end{array}\right)\right\}$ and since these sets of matrices generate $\mathrm{GL}(2, \mathrm{Z})$ and $\mathrm{z}_{4}$ respectively then $\Phi\left(\tilde{\pi}_{0}\left(M_{p, q}\right)\right) \approx Z_{4} \oplus \mathrm{GL}(2, \mathrm{z})$. Combining Propositions 2.5 and 2.6, we obtain Theorem 2.1 (ii).

REMARK. If $p$ is odd but $\neq 1,3,7$ and $q$ is even, we get the same result as in Proposition 2.5 using equivalent method. Also if $p=1,3,7$ and $q$ is even, we obtain the same result as that of Proposition 2.6.

Since $M_{p}^{+}, q$ denotes the subgroup of $M_{p, q}$ consisting of diffeomorphisms of $s^{p} \times s^{q} \# s^{p} \times s^{q}$ which induce identity map on all homology groups, it follows that $\mathrm{M}_{\mathrm{p}, \mathrm{q}}^{+}$is the kernel of the homomorphism $\Phi$. We now compute $\mathrm{M}_{\mathrm{p}, \mathrm{q}}^{+}$. We define a homomorphism

$$
\mathrm{G}: \tilde{\pi}_{0}\left(\mathrm{M}_{\mathrm{p}, \mathrm{q}}^{+}\right) \rightarrow \pi_{\mathrm{p}} \mathrm{SO}(\mathrm{q}+1)
$$

Given an element $\{f\} \in \tilde{\pi}_{0}\left(M_{p, q}^{+}\right)$, since $\Phi(f)$ is identity, it means that if $i\left(s^{p} \times\left\{p_{0}\right\}\right)$ is the usual identity embedding of $s^{p} \times\left\{p_{0}\right\}$ into $s^{p} \times s^{q} \# s^{p} \times s^{q}$ where $p_{0}$ is a fixed point in $s^{q}$ far away from the connected sum, then the sphere $s^{p} \times\left\{p_{0}\right\}$ in $s^{p} \times s^{q} \# s^{p} \times s^{q}$ represents a generator of the homology

$H_{p}\left(S^{p} \times S^{q} \# S^{p} \times S^{q}\right) \approx z \oplus z$. Since $\Phi(f)$ is identity, it follows that $f\left(s^{p} \times p_{0}\right)$ is homologous to $i\left(S^{p} \times p_{0}\right)$ and since $p<q$ and by Hurewicz theorem, $f$ and $i$ are homotopic and in fact with the dimension restriction, they are diffeotopic. By tubular neighborhood theorem, $f$ is diffeotopic to a map say $f^{\prime \prime}$ such that $f^{\prime \prime}\left(S^{p} \times D^{q}\right)=S^{p} \times D^{q}$ where $f^{\prime \prime}(x, y)=\left(x, \alpha\left(f^{\prime \prime}\right)(x) \cdot y\right)$ and $\alpha\left(f^{\prime \prime}\right): s^{p} \rightarrow s 0(q)$. Let $i: S O(q) \rightarrow S O(q+1)$ be the inclusion map and $i_{*}: \pi_{p} S O(q) \rightarrow \pi_{p} s O(q+1)$ the induced map on the homotopy groups. Then we define

$$
G\{f\}=i_{\star}\left(\alpha\left(f^{\prime \prime}\right)\right)
$$

LEMMA 2.7 G is wel1-defined.

PROOF: Let $f, h \in \mathrm{M}_{\mathrm{p}, \mathrm{q}}^{+}$such that $\mathrm{f}$ and $\mathrm{h}$ are pseudo-diffeotopic then $f \cdot h^{-1} \in M_{p, q}^{+}$is pseudo-diffeotopic to the identity. If $G\{f\}=i_{\star} \alpha\left(f^{\prime \prime}\right)$ and 
$G(h)=i_{*} \alpha\left(h^{\prime \prime}\right)$ where $f(x, y)=\left(x, \alpha\left(f^{\prime \prime}\right)(x) \cdot y\right)$ and $h(x, y)=\left(x, \alpha\left(h^{\prime \prime}\right)(x) \cdot y\right)$ for $(x, y) \in s^{p} \times D^{q}$ then it follows that

$$
f \cdot h^{-1}(x, y)=\left(x, \alpha\left(f^{\prime \prime}\right) \alpha\left(h^{\prime \prime}\right)^{-1}(x) \cdot y\right) \quad(x, y) \in s^{p} \times D^{q} .
$$

We wish to show that $i_{*} \alpha\left(f^{\prime \prime}\right)=i_{*} \alpha\left(h^{\prime \prime}\right)$. Since $G(f)=i_{*} \alpha\left(f^{\prime \prime}\right) \in \pi_{p}$ so $(q+1)$ and $G(h)=i_{*} \alpha\left(h^{\prime \prime}\right) \in \pi_{p} s 0(q+1)$ then we can define maps $f_{1}, h_{1} \in \operatorname{Diff}\left(s^{p} \times s^{q}\right)$ thus $f_{1}(x, y)=\left(x, i_{*} \alpha\left(f^{\prime \prime}\right)(x) \cdot y\right)$ and $h_{1}(x, y)=\left(x, i_{*} \alpha\left(h^{\prime \prime}\right)(x) \cdot y\right)$ then consider $f_{1} h_{1}^{-1} \in$ $\operatorname{Diff}\left(s^{p} \times s^{q}\right)$ defined by $f_{1} h_{1}^{-1}(x, y)=\left(x, i_{*} \alpha\left(f^{\prime \prime}\right) i_{*} \alpha\left(h^{\prime \prime}\right)^{-1}(x) \cdot y\right)(x, y) \in s^{p} \times s^{q}$. Since $f \cdot h^{-1}$ is pseudo-diffeotopic to identity so is $f_{1} \cdot h_{1}^{-1}$ by its definition. Hence $f_{1} \cdot h_{1}^{-1} \in \operatorname{Diff}\left(S^{p} \times S^{q}\right)$ is diffeotopic to the identity hence it extends to a diffeomorphism $g$ of $D^{p+1} \times S^{q}$, i.e., there exists $g \in \operatorname{Diff}\left(D^{p+1} \times S^{q}\right)$ such that g|Diff $\left(S^{p} \times S^{q}\right)=f_{1} \cdot h_{1}^{-1}$. Let $s_{\beta}$ denote the q-sphere bundle over $p+1-s$ phere with characteristic map $\beta: S^{p} \rightarrow$ so $(q+1)$. Then we have

$$
s i_{*} \alpha\left(f^{\prime \prime}\right) i_{*} \alpha\left(h^{\prime \prime}\right)^{-1}=D^{p+1} \times s^{q} \bigcup_{f_{1} h_{1}^{-1}} D^{p+1} \times s^{q}
$$

so this gives a q-sphere bundle over a p+1-sphere with the characteristic class of the equivalent plane bundle being $i_{*} \alpha\left(f^{\prime \prime}\right) \cdot i_{*} \alpha\left(h^{\prime \prime}\right)^{-1}$. However, $f_{1} h_{1}^{-1}$ extends to $g \in \operatorname{Diff}\left(D^{p+1} \times s^{q}\right)$ then we have

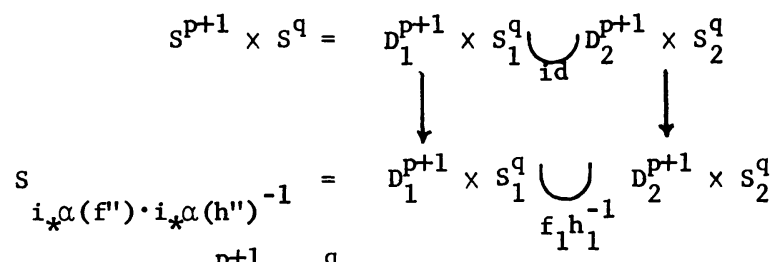

Hence we define a map $\mathrm{H}: \mathrm{s}^{\mathrm{p}+1} \times \mathrm{s}^{\mathrm{q}} \rightarrow \mathrm{s}$ i $_{*} \alpha\left(f^{\prime \prime}\right) \cdot i_{*} \alpha\left(h^{\prime \prime}\right)^{-1}$

$$
H(x, y)=\left\{\begin{array}{cll}
(x, y) & \text { if } & (x, y) \in D_{2}^{p+1} \times s_{2}^{q} \\
g(x, y) & \text { if } & (x, y) \in D_{1}^{p+1} \times s_{1}^{q}
\end{array} .\right.
$$

$H$ is we11-defined and is a diffeomorphism. This means that $s_{i_{*} \alpha\left(f^{\prime \prime}\right) \cdot i_{*} \alpha\left(h^{\prime \prime}\right)}-1$ is a trivial $\mathrm{q}-\mathrm{sphere}$ bundle over $\mathrm{s}^{\mathrm{p}+1}$ with characteristic class ${ }^{i_{*} \alpha\left(f^{\prime \prime}\right)^{*} i_{*} \alpha\left(\mathrm{h}^{\prime \prime}\right)^{-1}}$. It then follows from $[1$, Lemma $3.6(b)]$ that $i_{*} \alpha\left(f^{\prime \prime}\right)=i_{*}^{\alpha}\left(h^{\prime \prime}\right)$. Hence $G$ is we11defined. It is easy to see that $G$ is a homomorphism.

LEMMA $2.8 \quad G\left(\tilde{\pi}_{0}\left(M_{p, q}^{+}\right)\right)=i_{*}\left(\pi_{p}(S O(q))\right)$.

PROOF: By the definition of $G, G\left(\tilde{\pi}_{0}\left(M_{p, q}^{+}\right)\right) \subset i_{*}\left(\pi_{p} s O(q)\right)$ we then show that $i_{*}\left(\pi_{p} S O(q)\right) \subset G\left(\pi_{0}\left(M_{p, q}^{+}\right)\right)$. If $\alpha \in i_{*} \pi_{p}(S O(q))$ and $\{a\}=\alpha$ where a:s $\rightarrow$ so $(q+1)$ then we can define $f \in M_{p, q}$ by

$$
f(x, y)= \begin{cases}(x, a(x) \cdot y) & \text { if }(x, y) \in\left(s^{p} \times s^{q}\right)_{1} \\ (x, y) & \text { if }(x, y) \in\left(s^{p} \times s^{q}\right)_{2}\end{cases}
$$

since $a \in i_{*}(\pi(S O(q)))$ then $f \in \tilde{\pi}_{0}\left(M_{p, q}^{+}\right)$and so $G(f)=\alpha \in i_{*}\left(\pi_{p} S O(q)\right)$.

In fact since $p<q$, then $\pi_{p}\left(s^{q}\right)=0$ hence it follows from the exact sequence $\pi_{p+1} s^{q} \rightarrow \pi_{p} S_{q} \stackrel{i_{*}}{\longrightarrow} \pi_{p} S_{q+1} \rightarrow \pi_{p} s^{q}-\cdots>$ that $i_{*}$ is an epimorphism and so it is easily seen that $G$ is surjective. Hence the proof. 
The next lemma is similar to [6, Lemma 3.3].

LEMMA 2.9 Let $u \in \operatorname{ker} G$, then there exists a representative $f \in M_{p, q}^{+}$of $u$ such that $f$ is identity on $S^{p} \times D^{q}$.

PROOF: If $p<q-1$, then $\pi_{p+1}\left(s^{q}\right)=0$ and also $\pi_{p}\left(s^{q}\right)=0$ and so it follows from the exact sequence

$$
\rightarrow->\pi_{p+1}\left(\mathrm{~S}^{q}\right) \rightarrow \pi_{p}(\mathrm{SOq}) \stackrel{i_{\star}}{\rightarrow} \pi_{\mathrm{p}}(\mathrm{SOq}+1) \rightarrow \pi_{\mathrm{p}}\left(\mathrm{S}^{\mathrm{q}}\right) \rightarrow->
$$

that $i_{*}$ is an isomorphism hence if $u=\{f\} \in \operatorname{ker} G$ then $G(u)=i_{*} \alpha\left(f^{\prime \prime}\right)=0$ implies $\alpha\left(f^{\prime}\right)=0$. Since $f(x, y)=\left(x, \alpha\left(f^{\prime}\right)(x) \cdot y\right)$ for $(x, y) \in s^{p} \times D^{q}$ then it means $f(x, y)=(x, y)$ hence $f$ is identity on $s^{p} \times D^{q}$. However, in general let $g \in M_{p, q}$ be defined thus, if $S^{p} \times D_{+}^{q}, S^{p} \times D_{-}^{q}$ are subsets of $\left(S^{p} \times S^{q}\right)_{1}$, away from the connected sum in $M_{p, q}$, we then define

$$
g(x, y)=\left\{\begin{array}{l}
\left(x, \alpha\left(f^{\prime \prime}\right)^{-1}(x) \cdot y\right) \text { for }(x, y) \in s^{p} \times D_{+}^{q} \text { and } s^{p} \times D_{-}^{q} \subset\left(s^{p} \times s^{q}\right)_{1} \\
(x, y) \quad\left(s^{p} \times s^{q}\right)_{2}
\end{array}\right.
$$

since $i_{*} \alpha\left(f^{\prime \prime}\right) \in \pi_{p}($ So $(q+1))$ we define $g^{\prime} \in M_{p, q}$ by

$$
g^{\prime}(x, y)=\left\{\begin{array}{lll}
\left(x, i_{*} \alpha\left(f^{\prime \prime}\right)^{-1}(x) \cdot y\right) & \text { if }(x, y) \in\left(s^{p} \times s^{q}\right)_{1} \\
(x, y) & \text { if }(x, y) \in\left(s^{p} \times s^{q}\right)_{2}
\end{array}\right.
$$

then $g$ and $g^{\prime}$ are diffeotopic and since $u \in$ ker $G, G(u)=0=i_{\star} \alpha\left(f^{\prime \prime}\right)$ then $g^{\prime}$ is pseudo-diffeotopic to the identity and so follows that $g$ is also pseudodiffeotopic to the identity in $M_{p, q}$. Then the composition $g \circ f$ is pseudodiffeotopic to $f$ and clearly by the definition of $g, g \circ f$ keeps $s^{p} \times D_{+}^{q}$ fixed and represents $u$ because it is pseudo-diffeotopic to $f$. Hence the proof.

We now wish to compute $\operatorname{ker} G$. To do this, we define a homomorphism

$$
N: \operatorname{Ker} G \longrightarrow \tilde{\pi}_{0}\left(\operatorname{Diff}^{+}\left(S^{p} \times S^{q}\right)\right) \quad \text { and }
$$

show that $N$ is surjective. Here we adopt the notation $\operatorname{Diff}^{+}\left(S^{p} \times S^{q}\right)$ to mean the set of all diffeomorphisms of $s^{p} \times s^{q}$ to itself which induce identity on all homology groups. Given $u \in \operatorname{Ker} G$, let $f \in M_{p, q}^{+}$be $i$ ts representative then it follows from Lemma 2.9 that we can take $f$ to be identity on $S^{p} \times D^{q}$. So we have a map

$$
f:\left(s^{p} \times s^{q}\right)_{1} \#\left(s^{p} \times s^{q}\right)_{2} \rightarrow\left(s^{p} \times s^{q}\right)_{3} \#\left(s^{p} \times s^{q}\right)_{4} \text { such that }
$$

$f$ is identity on $S^{p} \times D^{q} \subset\left(S^{p} \times S^{q}\right)_{1}$.

Using the technique introduced by Milnor [9] and [3], we perform the spherical modification on the domain $\left(s^{p} \times s^{q}\right)_{1} \#\left(s^{p} \times s^{q}\right)_{2}$ that removes $s^{p} \times D^{q} \subset\left(s^{p} \times s^{q}\right)_{1}$ and replaces it with $\mathrm{D}^{\mathrm{p}+1} \times \mathrm{S}^{\mathrm{q}-1}$. Clearly we obtain $\left(\mathrm{S}^{\mathrm{p}} \times \mathrm{S}^{\mathrm{q}}\right)_{2}$ since $s^{p} \times D^{q} \bigcup_{i d} b^{p+1} \times s^{q-1}$ is diffeomorphic to $s^{p+q}$. Since $f$ is the identity on $s^{p} \times D^{q}$, we can assume that $f\left(S^{p} \times D^{q}\right)=s^{p} \times D^{q} \subset\left(s^{p} \times S^{q}\right)_{3}$ and then perform the corresponding spherical modification on the range $\left(s^{p} \times s^{q}\right)_{3} \#\left(s^{p} \times s^{q}\right)_{4}$ to obtain $\left(s^{p} \times s^{q}\right)_{4}$. After this modification we are then left with a diffeomorphism say $f^{\prime}$ of $\left(s^{p} \times s^{q}\right)_{1}$ onto $\left(s^{p} \times s^{q}\right)_{4}$, i.e., $f^{\prime} \in \operatorname{Diff}\left(s^{p} \times s^{q}\right)$ since $f \in M_{p, q}^{+}$then $f^{\prime} \in \operatorname{Diff}^{+}\left(s^{p} \times s^{q}\right)$. So we define $N\{f\}=\left\{f^{\prime}\right\}$. 
LEMMA $2.10 \mathrm{~N}$ is wel1-defined.

PROOF: Let $f, g \in \operatorname{Ker} G$ such that $f$ is pseudo-diffeotopic to $g$, then $f$ is identity on $S^{P} \times D^{q}$ and $g$ is also identity on $S^{P} \times D^{q}$. Since $f$ is pseudodiffeotopic to $g$ then there exists a diffeomorphism

$F \in \operatorname{Diff}\left(\left(S^{p} \times S^{q} \# S^{p} \times S^{q}\right) \times I\right)$ such that $F$ is identity on $S^{p} \times D^{q} \times I$ and $F \mid\left(S^{p} \times S^{q} \# S^{p} \times S^{q}\right) \times 0=f$ while $F \mid\left(S^{p} \times S^{q} \# s^{p} \times S^{q}\right) \times 1=g$. If we now perform the spherical modification on the domain $\left(s^{p} \times s^{q}\right)_{1} \#\left(s^{p} \times s^{q}\right)_{2} \times I$ of $F$ by removing $S^{p} \times D^{q} \times I \subset\left(S^{p} \times S^{q}\right)_{1} \times I$ and replacing it with $D^{p+1} \times S^{q-1} \times I$, then we obtain the manifold $\left(S^{p} \times S^{q}\right)_{2} \times I$ and since $F$ is identity on $S^{p} \times D^{q} \times I$, we then perform the corresponding modification on the range $\left(s^{p} \times S^{q}\right)_{3} \#\left(s^{p} \times s^{q}\right)_{4} \times I$ by removing $S^{p} \times D^{q} \times I \subset\left(S^{p} \times S^{q}\right)_{3} \times I$ and replacing it with $D^{p+1} \times S^{q-1} \times I$ to obtain $\left(S^{p} \times S^{q}\right)_{4} \times I$. We then obtain a diffeomorphism

$$
F^{\prime}:\left(S^{p} \times S^{q}\right)_{2} \times I \longrightarrow\left(S^{p} \times S^{q}\right)_{4} \times I
$$

i.e., $F^{\prime} \in \operatorname{Diff}^{+}\left(S^{p} \times S^{q} \times I\right)$ hence $N(F)=F^{\prime}$ and $F^{\prime} \mid\left(S^{p} \times S^{q} \times 0\right)=f^{\prime}$ and $F^{\prime} \mid S^{p} \times S^{q} \times 1=g^{\prime}$ hence $f^{\prime}$ is pseudo-diffeotopic to $g^{\prime}$ and so $N$ is well-defined. It is easy to see that $N$ is a homomorphism.

LEMMA 2.11 $\mathrm{N}$ is surjective.

PROOF: Let $h^{\prime} \in \operatorname{Diff}^{+}\left(S^{P} \times S^{q}\right)$, we need to find a diffeomorphism $h \in M^{+}$, such that $N(h)=h^{\prime}$. If $D^{p+q}$ is a disc in $s^{p} \times S^{q}$ then we can assume $h^{\prime}$ is identity on $D^{p+q}$ then we have $h^{\prime} \in \operatorname{Diff}^{+}\left(S^{p} \times S^{q}-D^{p+q}\right)$. We then define $h \in M_{p, q}^{+}$ thus

$$
h(x, y)=\left\{\begin{array}{lll}
(x, y) & \text { if }(x, y) \in\left(s^{p} \times s^{q}\right)_{1}-D^{p+q} \\
h^{\prime}(x, y) & \text { if }(x, y) \in\left(s^{p} \times s^{q}\right)_{2}-D^{p+q}
\end{array}\right.
$$

where $M_{p, q}^{+}=\operatorname{Diff}^{+}\left(S^{p} \times S^{q}\right)_{1} \#\left(S^{p} \times S^{q}\right)_{2}$ as earlier stated. $h$ is well-defined and $h \in M^{+}{ }^{*}$. Since $h$ is identity on $\left(S^{p} \times S^{q}\right)_{1}$ then it is identity on $S^{p} \times D^{q} \subset\left(S^{p} \times S^{q}\right)_{1}$ hence $h \in \operatorname{Ker} G$ and clearly $N(h)=h^{\prime}$ and so $N$ is surjective.

We recall from $[6,53]$ the homomorphism

$$
\text { B }: \tilde{\pi}_{0} \text { Diff }{ }^{+}\left(s^{p} \times s^{q}\right) \longrightarrow \pi_{p} S O(q+1) \text { which is similarly }
$$

defined as homomorphism $G$ and where Sato gave a computation of Ker B . We will apply this result of Ker $B$ to the next lemma.

LEMMA 2.12 Ker $\mathrm{N}$ is in one-to-one correspondence with Ker B.

PROOF: Let $f \in \operatorname{Ker} B$, we will produce a diffeomorphism $f^{\prime} \in M_{p, q}^{+}$such that $f^{\prime} \in \operatorname{Ker} N$. Since $f \in \operatorname{Ker} B$ then $f \in \operatorname{Diff}^{+}\left(s^{p} \times s^{q}\right)$ and $f \mid s^{p} \times D^{q}=$ identity. We define a diffeomorphism $f^{\prime}:\left(s^{p} \times s^{q}\right)_{1} \#\left(s^{p} \times s^{q}\right)_{2} \rightarrow\left(s^{p} \times s^{q}\right)_{3}{ }^{\#}\left(s^{p} \times s^{q}\right)_{4}$ by

$$
f^{\prime}(x, y)=\left\{\begin{array}{lll}
f(x, y) & \text { if } & (x, y) \in\left(s^{p} \times s^{q}\right)_{1}-D^{p+q} \\
(x, y) & \text { if } & (x, y) \in\left(s^{p} \times s^{q}\right)_{2}-D^{p+q}
\end{array}\right.
$$

$f^{\prime}$ is well-defined and $f^{\prime} \in M_{p, q}^{+}$. Since $f^{\prime}=f$ on $\left(S^{p} \times s^{q}\right)$, and since $f \mid s^{P} \times D^{q} \subset\left(S^{P} \times s^{q}\right)_{1}$ is identity then it follows that $f^{\prime} \mid S^{p} \times D^{q}=$ identity and so $f^{\prime} \in \operatorname{Ker} G$. However, using $s^{p} \times D^{q} \subset\left(s^{p} \times s^{q}\right)_{1}$ to perform spherical modification on both sides of the domain and range of $f^{\prime}$ and the fact that $f^{\prime}$ is the identity on $\left(S^{p} \times S^{q}\right)_{2}$ we clearly see that $N\left(f^{\prime}\right)=$ identity $\in \operatorname{Diff}\left(S^{p} \times S^{q}\right)_{2}$ hence $f^{\prime} \in \operatorname{Ker} N$. 
Converse1y let $f \in \operatorname{Ker} N$, then $N(f)=f^{\prime} \in \tilde{\pi}_{0} \operatorname{Diff}^{+}\left(S^{p} \times S^{q}\right)$. We want to show that $f^{\prime} \in \operatorname{Ker} B$. Since $f \in \operatorname{Ker} N$ then $i t$ means the image of $f$ under $N$ is trivial hence $N(f)=f^{\prime}$ is pseudo-diffeotopic to the identity. We now consider $B\left(f^{\prime}\right)$ where $B: \tilde{\pi}_{0} \operatorname{Diff}^{+}\left(S^{p} \times S^{q}\right) \rightarrow \pi_{p}(S O q+1)$ is defined in [6] similar to our homomorphism $G$. Since $f^{\prime} \in \operatorname{Diff}^{+}\left(S^{p} \times S^{q}\right)$ and $p<q$ then $f^{\prime} / s^{p} \times D^{q}=s^{p} \times D^{q}$ where $f^{\prime}(x, y)=\left(x, b\left(f^{\prime}\right)(x) \cdot y\right)$ for $(x, y) \in s^{p} \times D^{q}$ and $b\left(f^{\prime}\right): s^{p} \rightarrow s o(q)$. If $i: S O(q) \rightarrow S O(q+1)$ is the inclusion map and $i_{*}: \pi_{p} S O(q) \rightarrow \pi_{p} s O(q+1)$ is the induced homomorphism then $B\left(f^{\prime}\right)=i_{*} b\left(f^{\prime}\right) \in \pi_{p} s O(q+1)$.

However since $f^{\prime}$ is pseudo-diffeotopic to the identity then let $\mathrm{H}: \mathrm{S}^{\mathrm{P}} \times \mathrm{S}^{\mathrm{q}} \times \mathrm{I} \longrightarrow \mathrm{S}^{\mathrm{P}} \times \mathrm{S}^{\mathrm{q}} \times \mathrm{I}$ be the pseudo-diffeotopy between $\mathrm{f}^{\prime}$ and identity id . Then

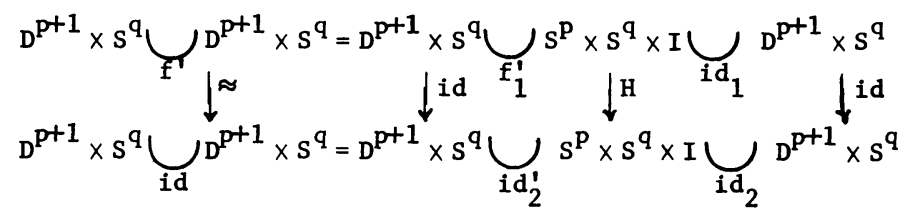

is the required diffeomorphism between $D^{p+1} \times S^{q} \bigcup_{f^{\prime}} D^{p+1} \times S^{q}$ and $D^{p+1} \times S^{q} \cup D^{p+1} \times S^{q}=$ $=\cdot \mathrm{s}^{\mathrm{p}+1} \times \mathrm{s}^{\mathrm{q}}$ where $\mathrm{id}_{1}(\mathrm{x}, \mathrm{y})=(\mathrm{x}, \mathrm{y}, 1), \mathrm{id}_{2}^{\prime}(\mathrm{x}, \mathrm{y}, 0)=(\mathrm{x}, \mathrm{y}), \mathrm{f}_{1}^{\prime}(\mathrm{x}, \mathrm{y}, 0)=\mathrm{f}^{\prime}(\mathrm{x}, \mathrm{y})$ and $\operatorname{id}_{2}(x, y)=i d(x, y, 1)=(x, y)$. However, consider $s_{i_{*} b\left(f^{\prime}\right)}$ the q-sphere bundle over a $(p+1)$-sphere whose characteristic class of the equivalent normal bundle is $i_{*} b\left(f^{\prime}\right) \in \pi_{p} s 0(q+1)$ hence $s_{i_{\star} b\left(f^{\prime}\right)}=D^{p+1} \times s^{q} \bigcup_{f^{\prime}} D^{p+1} \times s^{q} \approx s^{p+1} \times s^{q}$ by the above diffeomorphism and since $p<\stackrel{q}{q}$ it follows by $\left[f\right.$, Prop. 3.6] that $i_{\star} b\left(f^{\prime}\right)=0$. Hence $f^{\prime} \in \operatorname{Ker} B$ and so $\operatorname{Ker} N$ is in one-to-one correspondence with Ker B . Since $\mathrm{N}$ is surjective by Lemma 2.11 then we have

LEMMA 2.13 The order of the group Ker G equals the order of the direct sum group

$$
\operatorname{Ker} B \oplus \tilde{\pi}_{0} \operatorname{Diff}^{+}\left(s^{p} \times s^{q}\right)
$$

Also since $G$ is surjective by Lemma 2.8 then it is easily seen that

LEMMA 2.14 The order of $\tilde{\pi}_{0}\left(M_{\mathrm{p}, \mathrm{q}}^{+}\right)$is equal to the order of the direct sum group

$$
\pi_{p} s 0(q+1) \oplus \operatorname{Ker} B \oplus \pi_{0} D i f f^{+}\left(s^{p} \times s^{q}\right)
$$

However one can easily deduce from $[6,54]$

LEMMA $2.15 \quad \operatorname{ker} B \approx \pi_{q} S O(p+1) \oplus \theta^{p+q+1}$

Also from [6, Thm. II] and [1, Thm. 3.10] we have

LEMMA $2.16 \tilde{\pi}_{0} \operatorname{Diff}^{+}\left(\mathrm{s}^{\mathrm{p}} \times \mathrm{s}^{\mathrm{q}}\right)=\pi_{\mathrm{p}} \mathrm{so}(\mathrm{q}+1) \oplus \pi_{\mathrm{q}} \mathrm{so}(\mathrm{p}+1) \bullet \theta^{\mathrm{p}+\mathrm{q}+1}$

Combining Lemmas 2.12, 2.13, 2.14, 2.15, and 2.16, we obtain

THEOREM 2.17 For $p<q$, the order of the group $\tilde{\pi}_{0}\left(M_{p, q}^{+}\right)$equals twice the order of the group $\pi_{p} s O(q+1) \oplus \pi_{q} s 0(p+1) \oplus \theta^{p+q+1}$.

\section{CLASSIFICATION OF MANIFOLDS}

Consider the class of manifolds $\left\{M, \lambda_{1}, \lambda_{2}\right\}$ where $M$ is a manifold of type 
$(n, p, 2)$ where $n=p+q+1$ and $p=3,5,6,7(\bmod 8)$ and $\lambda_{1}, \lambda_{2}$ are the generators of $H_{p}(M)=z \oplus \mathbb{Z}$. By the proof of Theorem 1.1 we have an embedding $\varphi_{i}: S^{p} \times D^{q+1} \rightarrow M$ which represents the homology class $\lambda_{i} i=1,2$. If we then take the connected sum along the boundary of the two embedded copies of $S^{p} \times D^{q+1}$ we have an embedding

$$
\text { i : } s^{\mathrm{p}} \times \mathrm{D}^{\mathrm{q}+1} \# \mathrm{~S}^{\mathrm{p}} \times \mathrm{D}^{\mathrm{q}+1} \rightarrow \mathrm{M} \text { such that } i_{*}\left[\mathrm{~s}^{\mathrm{p}}\right]=\lambda_{1}+\lambda_{2}
$$

Two of such manifolds $\left\{M, \lambda_{1}, \lambda_{2}\right\}$ and $\left\{M^{\prime}, \lambda_{1}^{\prime}, \lambda_{2}^{\prime}\right\}$ will be said to be equivalent if there is an orientation preserving diffeomorphism of $M$ onto $M^{\prime}$ which takes $\lambda_{1}$ to $\lambda_{1}^{\prime} i=1,2$. Let $\mathscr{M}_{\mathrm{n}}$ be the equivalent class of manifolds satisfying these conditions. This equivalent class which is also the diffeomorphism class has a group structure. The operation is connected sum along the boundary $s^{p} \times s^{q} \# s^{p} \times s^{q}$ of $s^{p} \times D^{q+1} \# s^{p} \times D^{q+1}$. For if $\left\{M, \lambda_{1}, \lambda_{2}\right\},\left\{M^{\prime}, \lambda_{1}^{\prime}, \lambda_{2}^{\prime}\right\} \in \mathscr{M}_{n}$, then let $i_{1}: s^{p} \times D^{\mathrm{q}^{+1}} \# \mathrm{~s}^{\mathrm{P}} \times \mathrm{D}^{\mathrm{q}+1} \rightarrow \mathrm{M}$ be an orientation preserving embedding such that $i_{1 *}\left[s^{p}\right]=\lambda_{1}+\lambda_{2}$ and since there is an orientation reversing diffeomorphism of $\mathrm{s}^{\mathrm{P}} \times \mathrm{D}^{\mathrm{q}+1} \# \mathrm{~S}^{\mathrm{P}} \times \mathrm{D}^{\mathrm{q}+1}$ to itself (because $\mathrm{s}^{\mathrm{P}} \times \mathrm{D}^{\mathrm{q}+1}$ is a trivial $\mathrm{q}+1$-disc bundle over $\left.s^{p}\right)$ then we have an orientation reversing embedding $i_{2}: s^{p} \times D^{q+1} \# s^{p} \times D^{q+1} \rightarrow M^{\prime}$ such that $i_{2 *}\left[S^{P}\right]=\lambda_{1}^{\prime}+\lambda_{2}^{\prime}$. We now obtain $\underset{2 \mathrm{p}}{M M^{\prime}}$ from the disjoint sum $\left(M-\right.$ Int $\left.i_{1}\left(S^{p} \times D^{q+1} \# S^{p} \times D^{q+1}\right)\right) \cup\left(M^{\prime}-\right.$ Int $\left.i_{2}\left(S^{p} \times D^{q+1} \# s^{p} \times D^{q+1}\right)\right)$ by identifying $i_{1}(x)$ with $i_{2}(x)$ for $x \in s^{p} \times s^{q} \# s^{p} \times s^{q}$. We will call this operation the connected sum along double $p$-cycle. Where the $2 p$ in $M \# M^{\prime}$ means that we are identifying along the boundary of embedded copies of connected sum along the boundary of two copies of $S^{p} \times D^{q+1}$. It is easy to see that $H_{p}\left(M_{2 p}^{\#} M^{\prime}\right) \approx z \oplus Z$. Since we have identified $i_{1}\left(s^{p} \times s^{q} \# s^{p} \times s^{q}\right)$ with $i_{2}\left(s^{p} \times s^{q} \# s^{p} \times s^{q}\right)$ we can define $i_{1 *}\left[s^{P}\right]=\lambda_{1} \# \lambda_{1}^{\prime}+\lambda_{2} \# \lambda_{2}^{\prime}$ the generators of $H_{p}\left(M \# M^{\prime}\right)$ then we see that $M_{2 p} M^{\prime} \in \mathscr{L}_{n} \cdot$

LEMMA 3.1 The connected sum along the double p-cycle is we11-defined and associative.

PROOF: We need to show that the operation does not depend on the choice of the embeddings. Suppose there is another embedding $\varphi_{i}^{\prime}: s^{p} \times D^{q+1} \rightarrow M$ which represents the homology class $\lambda_{i} i=1,2$ and gives a corresponding embedding $i_{1}^{\prime}: s^{p} \times D^{q+1} \# s^{p} \times D^{q+1} \rightarrow M$. By the tubular neighborhood theorem $\varphi_{i}\left(s^{p} \times D^{q+1}\right)$ and $\varphi_{i}^{\prime}\left(S^{\mathrm{P}} \times \mathrm{D}^{\mathrm{q}+1}\right)$ differ only by rotation of their fiber, i.e., by an element of $\pi_{p} S O(q+1)=0$ since $p=3,5,6,7(\bmod 8)$ hence the two embeddings are isotopic and so the corresponding embeddings

$$
\begin{aligned}
& i_{1}: s^{p} \times D^{q+1} \# s^{p} \times D^{q+1} \longrightarrow M \quad \text { and } \\
& i_{1}^{\prime}: s^{p} \times D^{q+1}{ }_{\partial}^{\partial} s^{p} \times D^{q+1} \longrightarrow M \quad \text { are isotopic. }
\end{aligned}
$$

The definition does not therefore depend on the choice of $i_{1}$. With similar argument it does not depend on $i_{2}$. The connected sum is therefore well-defined. Associativity is easy to check. 
LEMMA 3.2 If $\left\{M, \lambda_{1}, \lambda_{2}\right\},\left\{M_{1}, \lambda_{1_{1}}, \lambda_{1_{2}}\right\} \in \mathscr{M}_{\mathrm{n}}$, such that they are equivalent.

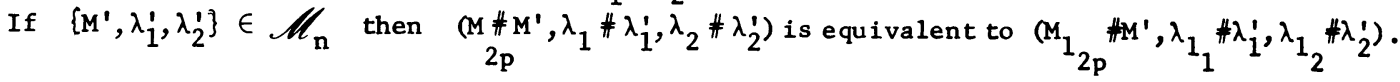

PROOF: Since $\mathrm{M}, \mathrm{M}_{1}$ are equivalent in $\mathscr{M}_{\mathrm{n}}$ then there exists an orientation preserving diffeomorphism $f: M \longrightarrow M_{1}$ which carries $\lambda_{1}$ to $\lambda_{1_{1}}$ and $\lambda_{2}$ to $\lambda_{1}$ hence it carries the embedding $\varphi_{i}\left(S^{p} \times D^{q+1}\right)$ to the corresponding embedding $\varphi_{1}\left(s^{p} \times D^{q+1}\right) i=1,2$ and so $f$ carries the embedding $i\left(s^{p} \times D^{q+1} \# s^{p} \times D^{q+1}\right) \subset M$ to the embedding $i_{1}\left(S^{p} \times D^{q+1} \# S^{p} \times D^{q+1}\right) \subset M_{1}$ hence $f$ induces a diffeomorphism

$$
f^{\prime}: M-\text { Int } i\left(s^{p} \times D^{q+1} \# s^{p} \times D^{q+1}\right) \rightarrow M_{1}-\text { Int } i_{1}\left(s^{p} \times D^{q+1} \# s^{p} \times D^{q+1}\right)
$$

which carries $\lambda_{1}$ to $\lambda_{1}$ and $\lambda_{2}$ to $\lambda_{1}$.

Trivially we have the identity map

id: $M^{\prime}$ - Int $i^{\prime}\left(S^{p} \times D^{q+1} \# S^{p} \times D^{q+1}\right) \rightarrow M^{\prime}-$ Int $i\left(S^{p} \times D^{q+1} \# S^{p} \times D^{q+1}\right)$

which carries $\lambda_{1}^{\prime}$ to $\lambda_{1}^{\prime}$ and $\lambda_{2}^{\prime}$ to $\lambda_{2}^{\prime}$. We then take the connected sum along their boundary $s^{p} \times s^{q} \# s^{p} \times s^{q}$ to have $M \# M^{\prime}$ which is disjoint sum of

M-Int $i\left(s^{p} \times D^{q+1} \# s^{p} \times D^{q+1}\right) \cup M^{\prime}-$ Int $i^{\prime}\left(s^{p} \times D^{q+1} \# s^{p} \times D^{q+1}\right)$ by identifying $i(x)$ and $i^{\prime}(x)$ for $x \in s^{\partial} \times s^{q} \# s^{p} \times s^{q}$. Similarly $M_{12 p}^{\#} M^{\prime}$ is the disjoint sum of

M-Int $i_{1}\left(s^{p} \times D^{q+1} \# s^{p} \times D^{q+1}\right) \cup M^{\prime}-$ Int $i^{\prime}\left(s^{p} \times D^{q+1}{ }_{\#} s^{p} \times D^{q+1}\right)$ by identifying $i_{1}(x)$ and $i^{\prime}(x)$ for $x \in s^{p} \times s^{q} \# s^{p} \times s^{q}$. Clearly we have a diffeomorphism $g: M_{2 p} M^{\prime} \rightarrow M_{1} \# M^{\prime}$ which is $f^{\prime}$ on $M$ and identity of $M^{\prime}$ and $g$ carries

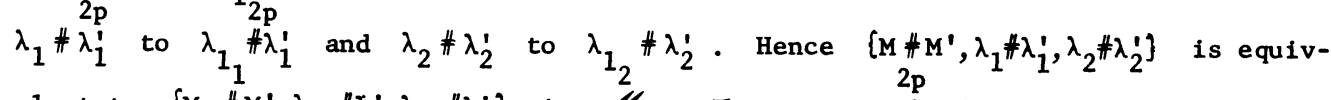
alent to $\left\{M_{1}{ }_{2 p}^{\# M^{\prime}, \lambda_{1}} \# \lambda_{1}^{\prime}, \lambda_{1} \# \lambda_{2}^{\prime}\right\}$ in $\mathscr{M}_{\mathrm{n}}$. That proves the lemma.

If we now take two copies of $S^{p} \times D^{q+1} \# s^{p} \times D^{q+1}$ and identify the two copies on $\partial$ their common boundaries by the identity map, we will obtain the manifold $s^{p} \times s^{q+1} \# s^{p} \times s^{q+1}$, i.e., $s^{p} \times s^{q+1} \# s^{p} \times s^{q+1}=\left(s^{p} \times D^{q+1} \# s^{p} \times D^{q+1}\right) \cup\left(s^{p} \times D^{q+1} \# s^{p} \times D^{q+1}\right)$ where id=identity: $s^{p} \times s^{q} \# s^{p} \times s^{q} \rightarrow s^{p} \times s^{q} \# s^{p} \times s^{q}$. If $\lambda_{0_{1}}, \lambda_{0_{2}}$ are the generators of $\mathrm{H}_{\mathrm{p}}\left(\mathrm{S}^{\mathrm{p}} \times \mathrm{S}^{\mathrm{q}+1_{\#}} \mathrm{~s}^{\mathrm{p}} \times \mathrm{s}^{\mathrm{q}+1}\right)=\mathrm{z} \bullet \mathrm{Z}$ and $-\lambda_{1}+\left(-\lambda_{2}\right) \in \mathrm{H}_{\mathrm{p}}(-\mathrm{M})=\mathbf{z} \bullet \mathrm{z}$ where $i_{*}\left[S^{P}\right]=-\lambda_{1}+-\lambda_{2}$ and $i: M \rightarrow-M$ is the orientation reversing diffeomorphism then we have the following.

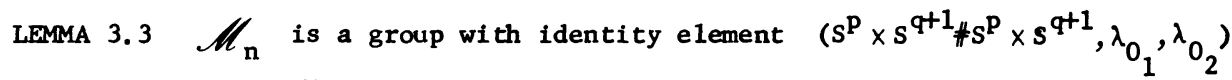
and for $\left(M, \lambda_{1}, \lambda_{2}\right) \in \mathscr{M}_{\mathrm{n}}\left(-M_{1},-\lambda_{1},-\lambda_{2}\right)$ is the inverse element.

To be able to prove our main theorem later, we need to investigate $\tilde{\pi}_{0} D_{i f f}^{+}\left(S^{p} \times D^{q+1} \# s^{p} \times D^{q+1}\right)$. As in the case of $\tilde{\pi}_{0}\left(M_{p, q}\right)$, we define a homomorphism $\Phi^{\prime}: \tilde{\pi}_{0} \operatorname{Diff}\left(\mathrm{s}^{\mathrm{p}} \times \mathrm{D}^{\mathrm{q}+1_{\# S^{\mathrm{P}}}} \times \mathrm{D}^{\mathrm{q}+1}\right) \rightarrow$ Auto $\mathrm{H}_{*}\left(\mathrm{~s}^{\mathrm{p}} \times \mathrm{D}^{\mathrm{q}+1_{\# S^{\mathrm{p}}}} \times \mathrm{D}^{\mathrm{q}+1}\right)$ by induced automorphism of homology groups. Since $s^{p} \times D^{q+1} A^{p} \times D^{q+1}$ has the homotopy type of $s^{p} \times D^{q+1}$ v $s^{p} \times D^{q+1}$ then

$$
H_{i}\left(S^{p} \times D^{q+1} \# S^{p} \times D^{q+1}\right)=\left\{\begin{array}{cl}
z & \text { if } i=0 \\
z \oplus z & \text { if } i=p
\end{array} .\right.
$$


Using similar ideas in $\$ 2$, it is easy to prove the following.

LEMMA 3.4

$$
\left.\Phi^{\prime} \tilde{\pi}_{0}\left(\operatorname{Diff}\left(S^{p} \times D^{q+1}{ }_{\#} S^{p} \times D^{q+1}\right)\right)\right)= \begin{cases}Z_{4} & \text { if } p \text { is even } \\ G L(2, Z) & \text { if } p=1,3,7 \\ H & \text { if } p \text { is odd but } \neq 1,3,7\end{cases}
$$

Let $\operatorname{Diff}{ }^{+}\left(S^{p} \times D^{q+1} \# s^{p} \times D^{q+1}\right) \subset \operatorname{Diff}\left(S^{p} \times D^{q+1} \# s^{p} \times D^{q+1}\right)$ be the set of all diffeomorphisms of $S^{p} \times D^{q^{q}+1}{ }_{\# S^{p}} \times D^{q+1}$ which induce identity automorphisms on its homology. Then it follows that $\tilde{\pi}_{0} D_{i f f}{ }^{+}\left(s^{p} \times D^{q+1} \# s^{p} \times D^{q+1}\right)$ is the kernel of $\Phi^{\prime}$. We define a homomorphism

$$
G^{\prime}: \pi_{p} S O(q+1) \longrightarrow \tilde{\pi}_{0} \operatorname{Diff}^{+}\left(s^{p} \times D^{q+1} \underset{\partial}{\# s^{p}} \times D^{q+1}\right)
$$

If $\alpha \in \pi_{p}$ SOq+1 and $a=\{a\}$ then we define a map

by

$$
g_{a}: s^{p} \times D^{q+1}{ }_{\partial} s^{p} \times D^{q+1} \longrightarrow s^{p} \times D^{q+1}{ }_{\partial} s^{p} \times D^{q+1}
$$

$$
g_{a}(x, y)= \begin{cases}(x, a(x) \cdot y) & \text { for }(x, y) \in\left(s^{p} \times D^{q+1}\right)_{1} \\ (x, a(x) \cdot y) & \text { for }(x, y) \in\left(s^{p} \times D^{q+1}\right)_{2}\end{cases}
$$

$\mathrm{g}_{\mathrm{a}}$ is clearly well-defined and it is a diffeomorphism and since $g_{a}$ keeps $\mathrm{s}^{\mathrm{p}}$ fixed,

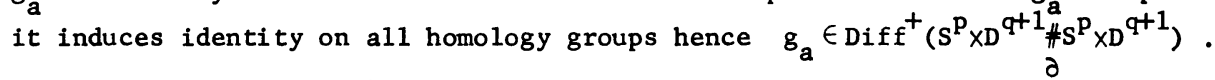

We will define $G^{\prime}\{\alpha\}=\left\{g_{a}\right\}$

LEMMA $3.5 G^{\prime}$ is well defined.

PROOF: If $a^{\prime} \in \pi_{p} S O(q+1)$ such that $a$ is homotopic to $a^{\prime}$ and let

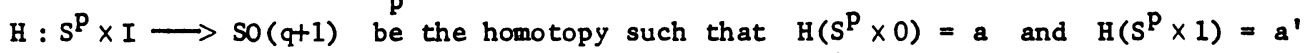
then we construct a diffeomorphism $F$ of $\left(S^{p} \times D^{q+1} \# S^{p} \times D^{q+1}\right) \times I$ by

$$
F(x, y, t)= \begin{cases}(x, H(x, t) \cdot y) & \left.(x, y) \in s^{p} \times D^{q+1}\right)_{1} \\ (x, H(x, t) \cdot y) & \left.(x, y) \in s^{p} \times D^{q+1}\right)_{2}\end{cases}
$$

This is the diffeotopy which connects $g_{a}$ and $g_{a^{\prime}}$.

LEMMA $3.6 \quad G^{\prime}$ is surjective.

PROOF: Let $\{f\} \in \tilde{\pi}_{0} \operatorname{Diff}^{+}\left(S^{p} \times D^{q+1} \# s^{p} \times D^{q+1}\right)$ then $f$ induces identity on all homology groups. However $H_{p}\left(S^{p} \times D^{q+1}{ }_{\partial} S^{\partial} \times D^{q+1}\right) \approx z \bullet z$ and so if $\lambda_{1}$ and $\lambda_{2}$ represents the generators of the first and second summand and the embeddings $i_{1}: s^{p} \times\left[p_{0}\right\} \longrightarrow s^{p} \times D^{q+1}{ }_{\pi} s^{p} \times D^{q+1}$ and $i_{2}: s^{p} \times\left\{p_{0}\right\} \longrightarrow s^{p} \times D^{q+1}{ }_{\pi} s^{p} \times D^{q+1}$ represents the homology class $\lambda_{1}$ and $\lambda_{2}$ respectively, since $f$ induces identity on homology then $f\left(S^{p} \times\left\{p_{0}\right\}\right.$ and $i_{1}\left(S^{p} \times\left\{p_{0}\right\}\right.$ are homologous. Since $p<q$ and by Hurewicz theorem $i_{1}$ and $f \circ i_{1}$ are homotopic, by Haefliger [10] and by the diffeotopy extension theorem and tubular neighborhood theorem, there exists $f^{\prime}$ in the diffeotopy class of $f$ such that $f^{\prime}(x, y)=(x, a(x) \cdot y)$ for $(x, y) \in\left(s^{p} \times D^{q+1}\right)_{1}$ where $s^{p} \times D^{q+1}$ is the tubular neighborhood of $s^{p} \times\left\{p_{0}\right\}$ and a: $s^{p} \rightarrow s_{q+1}$. Similar argument applies to the embedding $i_{2}: s^{p} \times\left\{p_{0}\right\} \rightarrow s^{p} \times D^{q+1_{\#}} s^{p} \times D^{q+1}$ and 
so we have a map $f^{\prime}$ in the diffeotopy class of $f$ hence in the diffeotopy class of $f^{\prime}$ and so $f^{\prime \prime}$ must be of the form $f^{\prime \prime}(x, y)=(x, a(x) \cdot y)$ where $(x, y) \in\left(s^{p} \times D^{q+1}\right)_{2}$. It follows that

$$
f(x, y)= \begin{cases}(x, a(x) \cdot y) & (x, y) \in\left(S^{p} \times D^{q+1}\right)_{1} \\ (x, a(x) \cdot y) & (x, y) \in\left(S^{p} \times D^{q+1}\right)_{2}\end{cases}
$$

Hence $G^{\prime}$ is surjective.

One can easily deduce from Lemma 3.6 that $\tilde{\pi}_{0} \operatorname{Diff}^{+}\left(S^{p} \times D^{q+1} \# S^{p} \times D^{q+1}\right)$ is a factor group of $\pi_{p}(\mathrm{SO} q+1)$.

THEOREM 3.7 Let $M$ be an $n$-dimensional closed simply connected manifold of type $(n, p, 2)$ where $n=p+q+1$ with $p=3,5,6,7$ (mod 8) then the number of differentiable manifolds satisfying the above conditions up to diffeomorphism is twice the order of the direct sum group $\pi_{p} s o(p+1) \oplus \theta^{n}$.

PROOF: We define a map $\mathrm{C}: \tilde{\pi}_{0}\left(\mathrm{M}_{\mathrm{p}, \mathrm{q}}^{+}\right) \longrightarrow \mathscr{M}_{\mathrm{n}}$ and show that $\mathrm{C}$ is an isomorphism. Let $\left\{\mathrm{f}^{*} \in \tilde{\pi}_{0}\left(\mathrm{M}_{\mathrm{p}, \mathrm{q}}^{+}\right)\right.$then $\mathrm{f}$ is a diffeomorphism of $\mathrm{s}^{\mathrm{p}} \times \mathrm{s}^{q_{\#}} \mathrm{~s}^{\mathrm{p}} \times \mathrm{s}^{\mathrm{q}}$ which induce identity on homology. We then take two copies $\left(S^{p} \times D^{q+1} \frac{1}{\partial} S^{p} \times D^{q+1}\right)_{1}$ and $\left.s^{p} \times D^{q+1} \# S^{p} \times D^{q+1}\right)_{2}$ of $S^{p} \times D^{q+1} \# s^{p} \times D^{q+1}$ and attach them on the boundary by $f$ to have $\left(S^{\frac{\partial}{p}} \times D^{q+1} \frac{\pi}{\partial} S^{p} \times D^{q+1}\right)_{1} \cup\left(S^{p} \times D^{q+1} \# S^{p} \times D^{q+1}\right)_{2}$. An orientation is chosen to be compatible with ${ }^{\partial}\left(S^{p} \times D^{q+1}{ }_{\# S^{p}}^{f} \times D^{q+1}\right)_{1}$ and the manifold obtained belongs to the group $\mathscr{M}_{\mathrm{n}}$. The generators of the $\mathrm{p}$-dimensional homology group is fixed to be the one represented by the usual embedding $s^{p} \times\left\{p_{0}\right\} \rightarrow\left(S^{p} \times D^{q+1}\right)_{1} \subset\left(s^{p} \times D^{q+1} \# s^{p} \times D^{q+1}\right)_{1}$ and $S^{p} \times\left\{p_{0}\right\} \rightarrow\left(s^{p} \times D^{q+1}\right)_{2} \subset\left(s^{p} \times D^{q+1} \# s^{p} \times D^{q+1}\right)_{1}$. We then define $\partial$ $c\{f\}=\left(S^{p} \times D^{q+1} \# S^{p} \times D^{q+1}\right) \cup\left(S^{p} \times D^{q+1} \# s^{p} \times D^{q+1}\right)$. We now show that $c$ is well-defined. Let $f_{0}, f_{1} \in M_{p, q}^{+} \quad$ such that $f_{0}$ is pseudo-diffeotopic to $f_{1}$ then there exists $H:\left(S^{p} \times S^{q}{ }^{q} S^{p} \times S^{q}\right) \times I \longrightarrow\left(S^{p} \times S^{q} \# S^{p} \times S^{q}\right) \times I$ such that $H(x, y, 0)=f_{0}$ and $H(x, y, 1)=f_{1}$ then we wish to show that $\left(S^{p} \times D^{q+1} \# s^{p} \times D^{q+1}\right) \cup\left(S^{p} \times D^{q+1} \# s^{p} \times D^{q+1}\right)$ is

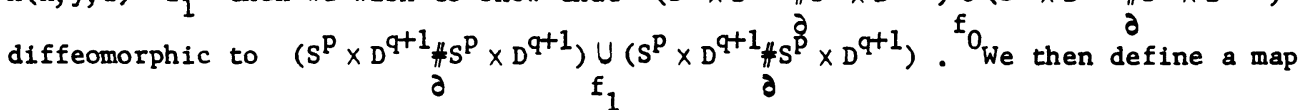

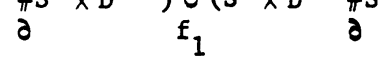

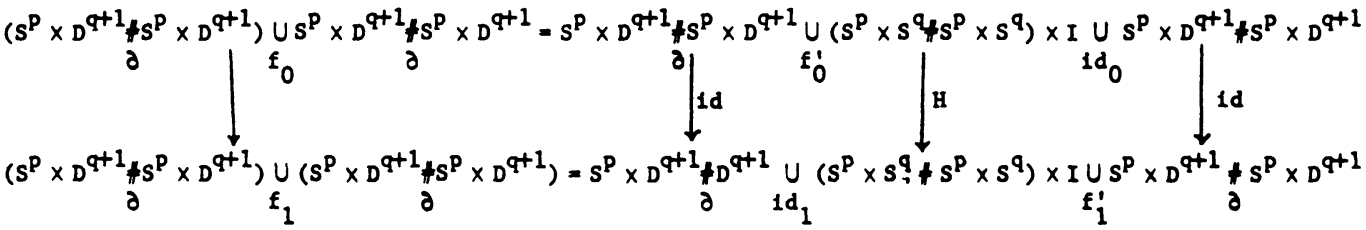

where $i d_{0}(x, y)=(x, y, 1), i d_{1}(x, y, 0)=(x, y), f_{0}^{\prime}(x, y, 0)=f_{0}(x, y)$ and $f_{1}^{\prime}(x, y)=f_{1}(x, y, 1)$. This is a well-defined map and is the required diffeomorphism from

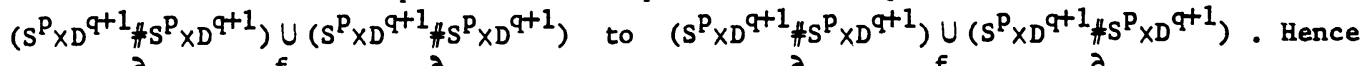
$C$ is well-defined and it is easy to see that $C$ is a homomorphism. By Theorem 1.1 it follows that $C$ is surjective. We now need to show that $C$ is injective. Suppose \{f $\in \tilde{\pi}_{0}\left(M_{p, q}^{+}\right)$and $C(f)=\left(M, \lambda_{1}, \lambda_{2}\right)$ is trivial, then it follows that 


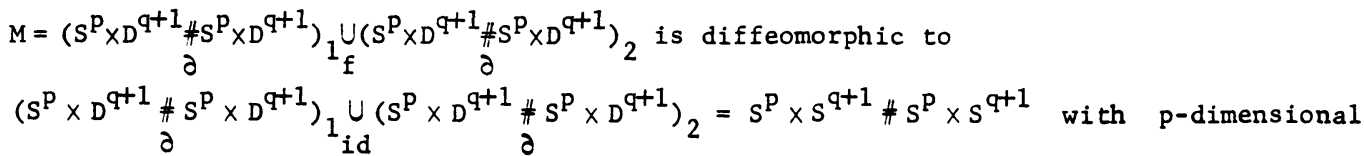
homology generators $\lambda_{0_{1}}, \lambda_{0_{2}}$, by a diffeomorphism $d$ which carries $\lambda_{1}$ to $\lambda_{0}$ and $\lambda_{2}$ to $\lambda_{0_{2}}$, i.e.,

$$
\begin{aligned}
& \left(S^{p} \times D^{q+1} \# S^{p} \times D^{q+1}\right){ }_{1} \underset{f}{U}\left(S^{p} \times D^{q+1} \# S^{p} \times D^{q+1}\right)_{2}
\end{aligned}
$$

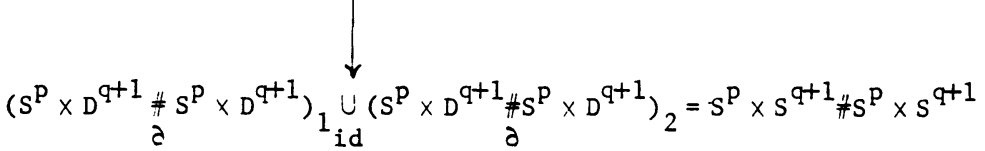

It is easy to see that since $d$ carries $\lambda_{1}$ to $\lambda_{0}$ and $\lambda_{2}$ to $\lambda_{0}$ and because $\mathrm{p}=3,5,6,7$ (mod 8$)$ then $\mathrm{d}$ is the identity on $\left(\mathrm{s}^{\mathrm{p}} \times \mathrm{D}^{\mathrm{q}+1} * \mathrm{~S}^{\mathrm{p}} \times \mathrm{D}^{\mathrm{q}+1}\right)_{1}$. On the boundary $\mathrm{S}^{\mathrm{p}} \times \mathrm{S}^{\mathrm{q}} \# \mathrm{~S}^{\mathrm{p}} \times \mathrm{s}^{\mathrm{q}}, \mathrm{d}$ is just $\mathrm{f}$. Since $\mathrm{d}$ is a diffeomorphism it follows that $f$ extends to a diffeomorphism of $\left(S^{p \times D^{q+1}} \# s^{p} \times D^{q+1}\right)_{2}$ which means $f \in \operatorname{Diff}^{+}\left(S^{p} \times S^{q} \# S^{p} \times S^{q}\right)$ is extendable to $\operatorname{Diff}^{+}\left(S^{p} \times D^{q+I} \# S^{p} \times D^{q+1}\right)$, but by Lemma 3.5, $\tilde{\pi}_{0} \operatorname{Diff}^{+}\left(\mathrm{s}^{\mathrm{p}} \times \mathrm{D}^{\mathrm{q}+1} \# \mathrm{~S}^{\mathrm{p}} \times \mathrm{D}^{\mathrm{q}+1}\right)$ is a factor group of $\pi_{\mathrm{p}}(\mathrm{SOq}+1 \hat{\rho}$ but since $p=3,5,6,7$, $\bmod 8$ then $\pi_{p}(S O q+1)=0$. Hence $f$ is pseudo-diffeotopic to the identity and so $C$ is injective. It then follows that $C$ is an isomorphism. By Theorem 2.17 and since $p=3,5,6,7(\bmod 8)$ it follows that the order of the group $\tilde{\pi}_{0}\left(M_{p, q}^{+}\right)$is twice the order of the group $\pi_{q} s O(p+1) \oplus \theta^{n}$ and since $C$ is an isomorphism the theorem is proved. The methods used here if carefully applied can be used to obtain a general resu1t.

THEOREM 3.8 If $M$ is a smooth, closed simply connected manifold of type $(n, p, r)$ where $n=p+q+1$ and $p=3,5,6,7(\bmod 8)$ then the number of differentiable manifolds up to diffeomorphism satisfying the above is equal to $r$ times the order of $\pi_{q} s O(p+1) \oplus \theta^{n}$.

\section{REFERENCES}

1. TURNER, E.C. Diffeomorphisms of a Product of Spheres, Inventiones Math. 8 , (1969) 69-82.

2. SATO, H. Diffeomorphism groups and classification of manifolds, J. Math. Soc. Japan, Vo1. 21, No. 1, (1969) 1-36.

3. MILNOR, J. and KERVAIRE, M. Groups of Homotopy Spheres I, Ann. of Math. $\underline{77}$ (1963) 504-537.

4. SMALE, S. On the structure of manifolds, Amer. J. Math. 84 (1962) 387-399.

5. KUROSH, A.G. Theory of Groups Vol. II, Chelsea, New York, 1955.

6. SATO, H. Diffeomorphism groups of $S^{p} \times S^{q}$ and Exotic Spheres, quart. J. Math., Qxford (2), 20 (1969) 255-276.

7. LEVINE, J. Self-Equivalences of $s^{n} \times s^{k}:$ Trans. American Math. Soc. 143 (1969) 523-543.

8. WALL, C.T.C. Killing the middle homotopy groups of odd dimensional manifolds, Trans. American Math. Soc. 103 (1962) 421-433.

9. MILNOR, J. A procedure for killing homotopy groups of differentiable manifolds, Proc. Symposium Tucson, Arizona, 1960.

10. HAEFLIGER, A. Plongements differentiables des variétés. Commentari Helvetici Math. 36, 47-82 (1961). 


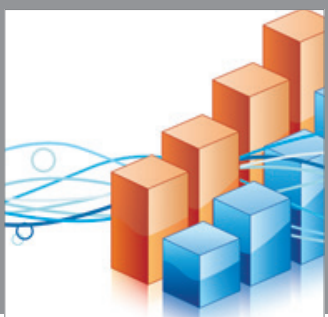

Advances in

Operations Research

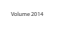

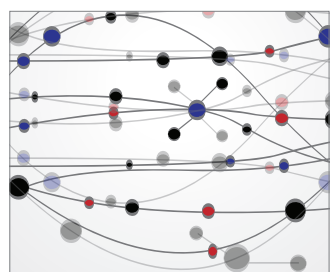

\section{The Scientific} World Journal
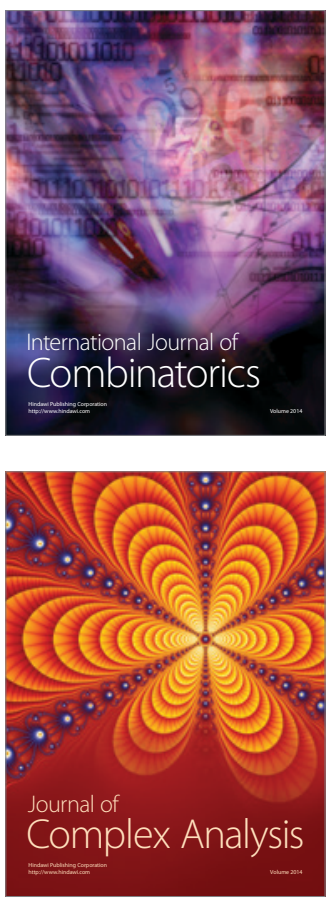

International Journal of

Mathematics and

Mathematical

Sciences
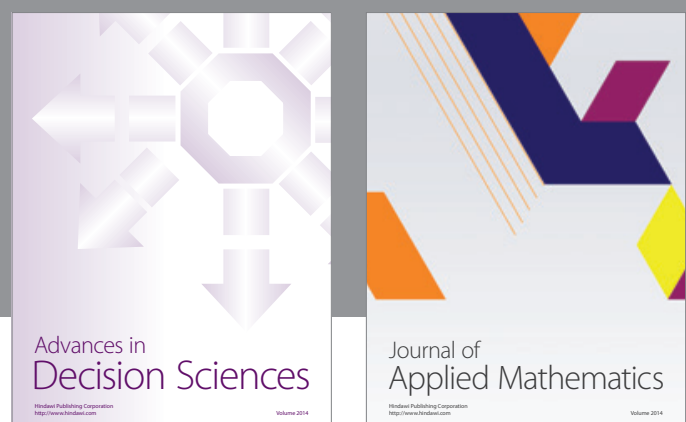

Journal of

Applied Mathematics
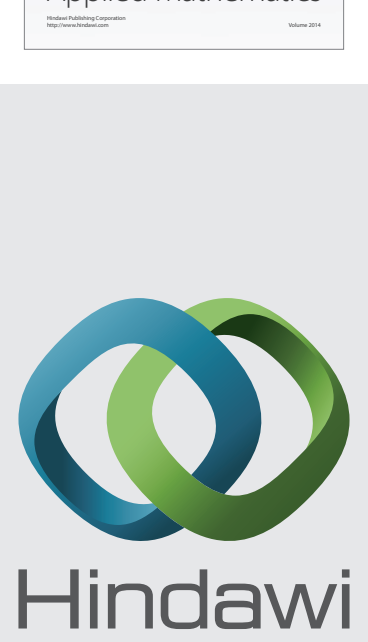

Submit your manuscripts at http://www.hindawi.com
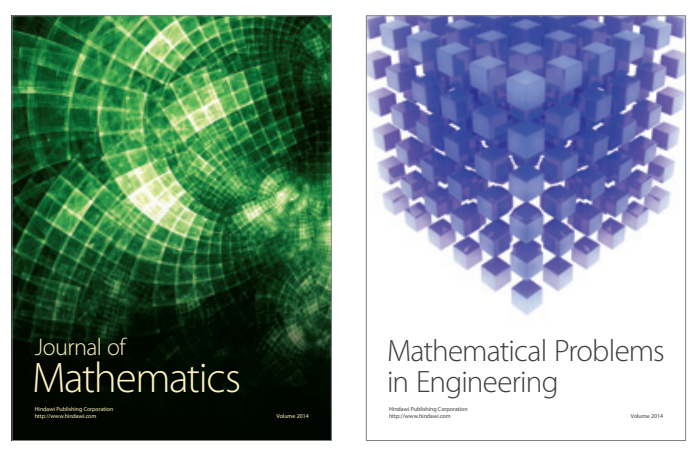

Mathematical Problems in Engineering
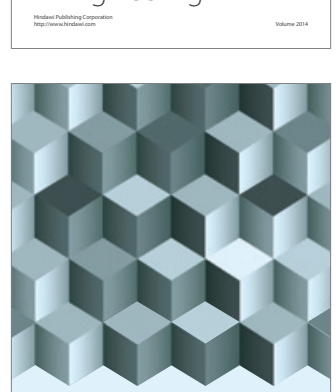

Journal of

Function Spaces
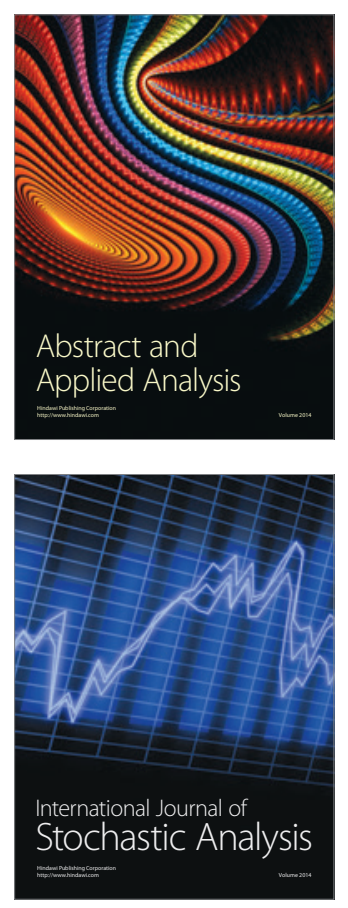

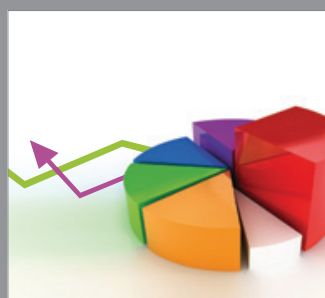

ournal of

Probability and Statistics

Promensencen
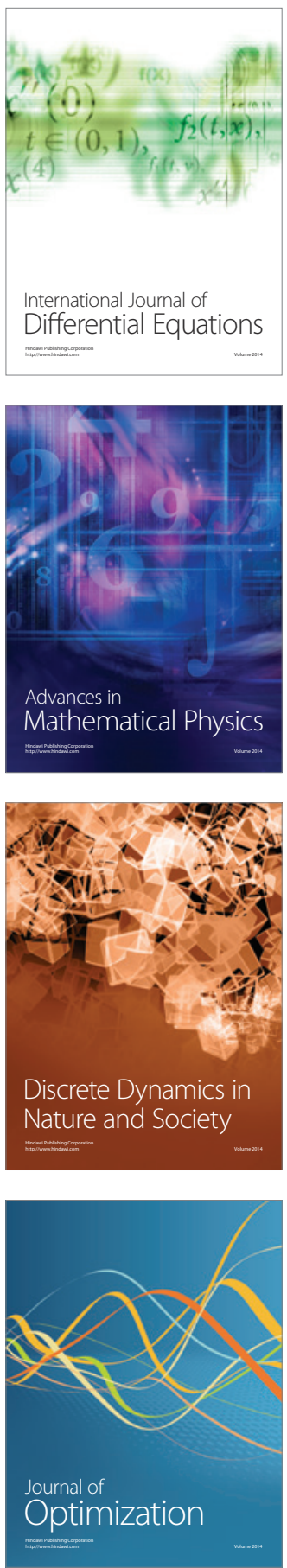